\title{
Paramagnetism and antiferromagnetic interactions in single-phase Fe-implanted $\mathrm{ZnO}$
}

\author{
L M C Pereira ${ }^{1,2,3}$, U Wahl ${ }^{3}$, J G Correia ${ }^{3}$, M J Van Bael ${ }^{4}$, K Temst ${ }^{1}$, \\ A Vantomme ${ }^{1}$ and J P Araújo ${ }^{2}$ \\ ${ }^{1}$ Instituut voor Kern- en Stralingsfysica, KU Leuven, B-3001 Leuven, Belgium \\ 2 IFIMUP and IN-Institute of Nanoscience and Nanotechnology, Universidade do Porto, \\ 4169-007 Porto, Portugal \\ ${ }^{3}$ Instituto Tecnológico e Nuclear, Instituto Superior Técnico, Universidade Técnica de Lisboa, \\ 2686-953 Sacavém, Portugal \\ ${ }^{4}$ Laboratory of Solid-State Physics and Magnetism, KU Leuven, B-3001 Leuven, Belgium \\ E-mail: lino.pereira@fys.kuleuven.be
}

Received 4 July 2013, in final form 9 August 2013

Published 11 September 2013

Online at stacks.iop.org/JPhysCM/25/416001

\begin{abstract}
As the intrinsic origin of the high-temperature ferromagnetism often observed in wide-gap dilute magnetic semiconductors becomes increasingly debated, there is a growing need for comprehensive studies on the single-phase region of the phase diagram of these materials. Here we report on the magnetic and structural properties of $\mathrm{Fe}$-doped $\mathrm{ZnO}$ prepared by ion implantation of $\mathrm{ZnO}$ single crystals. A detailed structural characterization shows that the $\mathrm{Fe}$ impurities substitute for $\mathrm{Zn}$ in $\mathrm{ZnO}$ in a wurtzite $\mathrm{Zn}_{1-x} \mathrm{Fe}_{x} \mathrm{O}$ phase which is coherent with the $\mathrm{ZnO}$ host. In addition, the density of beam-induced defects is progressively decreased by thermal annealing up to $900^{\circ} \mathrm{C}$, from highly disordered after implantation to highly crystalline upon subsequent annealing. Based on a detailed analysis of the magnetometry data, we demonstrate that isolated $\mathrm{Fe}$ impurities occupying $\mathrm{Zn}$-substitutional sites behave as localized paramagnetic moments down to $2 \mathrm{~K}$, irrespective of the Fe concentration and the density of beam-induced defects. With increasing local concentration of $\mathrm{Zn}$-substitutional Fe, strong nearest-cation-neighbor antiferromagnetic interactions favor the antiparallel alignment of the Fe moments.
\end{abstract}

(Some figures may appear in colour only in the online journal)

\section{Introduction}

The current view on wide-gap dilute magnetic semiconductors (DMS) is moving towards the belief that the often observed high-temperature ferromagnetism is not intrinsic, i.e. that it does not result from long-range magnetic order of randomly distributed magnetic dopants [1]. While limited experimental evidence exists that some DMS materials are intrinsically ferromagnetic at room temperature, a number of nonintrinsic sources of ferromagnetic-like behavior have been identified and are becoming increasingly well documented: magnetic contamination [2-5], instrumental artifacts [4-6] and chemical and/or structural segregation of the transition metal dopants into non-DMS phases [7-17].

Following the first report of high-temperature ferromagnetism in Co-doped $\mathrm{TiO}_{2}$ [18] and the prediction by Dietl et al [19] that highly p-type Mn-doped $\mathrm{ZnO}$ and $\mathrm{GaN}$ could attain a Curie temperature $\left(T_{\mathrm{C}}\right)$ above room temperature, ferromagnetism at and above room temperature has been reported in a rapidly growing number of wide-gap DMS materials (cf e.g. the reviews [20-23]). However, with the growing understanding of the most studied of these materials, issues of irreproducibility and instability became increasingly evident. Reports began to emerge which 


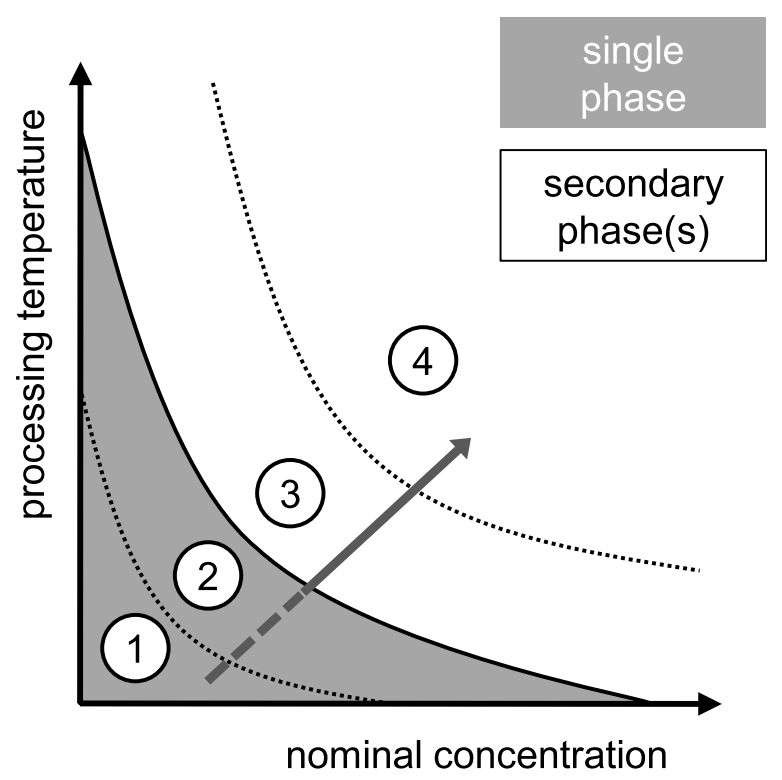

Figure 1. Representation of the structural phase diagram of a DMS system (described in detail in the text).

comprehensively described the magnetic behavior of some of these materials (e.g. [24-30]). Doing so requires covering growth, doping and post-processing conditions within relevant ranges, and performing both (1) magnetic characterization which carefully takes into account potential magnetic contamination and technique-specific artifacts, and (2) structural characterization which undeniably establishes either the single-phase character of the DMS material or identifies the segregated secondary phases. Such combined magnetostructural characterization, which typically implies using other than standard lab equipment (cf for example [30, 17]), then allows one to position a given DMS sample (in a given processing step) within the system's phase diagram.

Figure 1 illustrates the generic structural phase diagram for a DMS system consisting of a semiconductor $\left(\mathrm{A}_{y} \mathrm{~B}_{z}\right)$ doped with a transition metal $(\mathrm{X})$. The main coordinates in such a diagram are the processing temperature (of growth, implantation or annealing) and the nominal concentration (x) of the transition metal dopant, although the preparation method may also play an important role. The gray area represents the single-phase region, where the transition metal impurities substitute one of the host constituents (e.g. element A), maintaining the host's crystal structure by forming a $\mathrm{A}_{y-x} \mathrm{X}_{x} \mathrm{~B}_{z}$ alloy. The impurities may be randomly distributed (region (1)) or, for a sufficiently high concentration or temperature, aggregate in impurity-rich regions; i.e. $x$ may vary across the $\mathrm{A}_{y-x} \mathrm{X}_{x} \mathrm{~B}_{z}$ alloy without a well-defined interface (region (2)). For a sufficiently high processing temperature or concentration (white region), the transition metal impurities may segregate into small precipitates of a $\mathrm{A}_{y^{\prime}} \mathrm{X}_{x^{\prime}} \mathrm{B}_{z^{\prime}}$ (where $y^{\prime}$ and/or $z^{\prime}$ can be 0 ) secondary phase with a different structure from that of the host. Depending on various factors, such as processing temperature, impurity concentration and preparation method, different secondary phases may form (regions (3) and (4)).
The magnetic phase diagram of a DMS system can be pictured as an over-layer of the structural phase diagram of figure 1. In the true DMS region of the phase diagram (region (1)), the transition metal impurities are randomly and substitutionally distributed in the host. Intrinsic ferromagnetism can be defined as ferromagnetic order of the localized moments of these randomly distributed transition metal impurities via a long-range order mechanism, as is the case in narrow-gap DMS such as $\mathrm{Ga}_{1-x} \mathrm{Mn}_{x} \mathrm{As}$ and $\mathrm{In}_{1-x} \mathrm{Mn}_{x}$ As [1]. However, comprehensive studies on this region of the phase diagram of wide-gap DMS systems, on carefully characterized single-phase materials, revealed only paramagnetism (e.g. in Co-doped $\mathrm{ZnO}$ [25] and Mn-doped $\mathrm{GaN}$ [24]), antiferromagnetic interactions (e.g. in Co-doped $\mathrm{ZnO}$ [27, 28, 31, 32], Mn-doped $\mathrm{GaN}$ [26, 33], and $\mathrm{Cr}$ in $\mathrm{GaN}$ [34]), or at best, ferromagnetic order with a very low $T_{\mathrm{C}}$ (e.g. $T_{\mathrm{C}}<10 \mathrm{~K}$ in Mn-doped GaN [35]). With increasing processing temperature (either during growth, implantation or annealing), the impurities tend to aggregate and segregate. This segregation may be only chemical (region (2)), i.e. the crystal structure is maintained and only the local impurity concentration varies across the material, alternating between impurity-rich and impurity-poor regions (e.g. in Co-doped $\mathrm{ZnO}$ [7] and Mn-doped GaN [8]). For sufficiently high concentration and temperature, phase segregation may occur (regions (3) and (4)), i.e. the impurities precipitate in nanocomposites with a well-defined interface with the host structure (e.g. in $\mathrm{ZnO}$ doped with $\mathrm{Fe}, \mathrm{Co}$ and $\mathrm{Ni}$ [9-17]).

Fe-implanted $\mathrm{ZnO}$ is a rich DMS system in which to explore the different regions of such a complex magnetostructural phase diagram. The fact that the Fe impurities are incorporated by ion implantation offers the potential advantage that ion bombardment results in lattice disorder, which in turn has been proposed to promote ferromagnetic order of magnetic impurities in single-phase DMS materials, either in the form of point defects in bound magnetic polaron (BMP) models [36], or in the form of extended defects in charge transfer ferromagnetism (CTF) models [37]. In addition, Fe impurities may be incorporated in $\mathrm{ZnO}$ both as $\mathrm{Fe}^{2+}$ and $\mathrm{Fe}^{3+}$, making it a potentially suitable mixed-valency impurity in CTF systems. However, despite the various reports of intrinsic ferromagnetism in Fe-implanted $\mathrm{ZnO}$ (e.g. [38-41]), the extensive work by Zhou et al on carefully characterized samples has shown that the ferromagnetic-like behavior originates from superparamagnetic precipitates formed at sufficiently high processing temperatures and $\mathrm{Fe}$ concentrations: $\alpha$-Fe clusters (region (3) in figure 1) which are oxidized and then converted to the spinel ferrite $\mathrm{ZnFe}_{2} \mathrm{O}_{4}$ (region (4) in figure 1) with increasing annealing temperature and duration [9-12]. Although this segregated region of the phase diagram of Fe-implanted $\mathrm{ZnO}$ is rather well established (continuous part of the arrow in figure 1), both in terms of structure and magnetism, the magnetic behavior of the Fe impurities in single-phase $\mathrm{Zn}_{1-x} \mathrm{Fe}_{x} \mathrm{O}$ is still poorly understood (dashed part of the arrow in figure 1, i.e. regions (1) and (2)).

In this paper, we investigate the magnetism of singlephase $\mathrm{Zn}_{1-x} \mathrm{Fe}_{x} \mathrm{O}$ prepared by $\mathrm{Fe}^{+}$ion implantation in $\mathrm{ZnO}$ 
single crystals. By varying the nominal Fe concentration and the degree of structural disorder, we aim at identifying the type of magnetic interactions between the Fe moments and how it is affected by implantation-induced disorder.

\section{Experimental details}

Commercial $\mathrm{ZnO}$ wurtzite [0001] single crystals (CrysTec $\mathrm{GmbH}$ ), hydrothermally grown, were implanted with ${ }^{56} \mathrm{Fe}^{+}$ ions at room temperature to three different fluences: $1 \times 10^{15}$, $5 \times 10^{15}$ and $1 \times 10^{16}$ at. $\mathrm{cm}^{-2}$, referred to below as S1, S5 and S10 respectively. An implantation energy of $60 \mathrm{keV}$ and a beam angle of $10^{\circ}$ with respect to the sample surface (to minimize ion channeling) results in a peak atomic concentration $x_{\mathrm{p}}$ of $0.0068(0.68 \%), 0.034$ $(3.4 \%)$ and $0.068(6.8 \%)$, at a projected ion range $R_{\mathrm{p}}$ of $297 \AA$ (mean depth) with a straggling of $134 \AA$ (square root of the variance), estimated using MARLOWE [42]. In order to avoid sample contamination with ferromagnetic material [2], the implantations were carried out using a Mo sample-holder and the samples were placed in a ceramic boat during annealing. Three samples were prepared for each of the fluences: one for magnetic characterization using SQUID magnetometry, one for damage characterization using Rutherford backscattering and channeling spectrometry $(\mathrm{RBS} / \mathrm{C})$ and one for Fe lattice location using $\beta^{-}$emission channeling. Structural and magnetic characterization were performed in the as-implanted samples and after thermal annealing in vacuum $\left(<10^{-5}\right.$ mbar $)$ in $100^{\circ} \mathrm{C}$ steps $(10 \mathrm{~min}$ each step) up to $900^{\circ} \mathrm{C}$.

\subsection{SQUID magnetometry}

The magnetic characterization was performed using a superconducting quantum interference device (SQUID) magnetometer (Quantum Design MPMS XL-5) following strict procedures in order to avoid measurement artifacts and external magnetic contributions. These procedures were developed based on statistically relevant tests, which allowed us to determine the practical limits of SQUID magnetometry for the detection of ferromagnetism under various sample preparation, processing and handling conditions [2]. All measurements were performed with the field perpendicular to the $c$-axis, i.e., parallel to the sample plane.

\subsection{Rutherford backscattering and channeling spectrometry $(\mathrm{RBS} / \mathrm{C})$}

Defect accumulation and recovery were characterized using Rutherford backscattering and channeling spectrometry (RBS/C), with a $1.57 \mathrm{MeV} \mathrm{He}^{+}$beam and two detectors at backscattering angles of $168^{\circ}$ and $105^{\circ}$. The $168^{\circ}$ backscattering geometry was used to characterize the channeling minimum yield $\chi_{\min }$ along the [0001] axis. $\chi_{\min }$ is the ratio of the backscattering yield with the incident beam aligned with the crystal axis to the yield for a random beam incidence, and is a measure of the lattice disorder induced by ion implantation [43]. The $15^{\circ}$ glancing exit angle geometry (with respect to the sample surface) of the second detector was used to obtain enhanced depth resolution of the damage profile.

\section{3. $\beta^{-}$emission channeling}

The emission channeling (EC) technique allows one to determine the lattice location of impurities in single crystals, making use of the charged particles emitted by a radioactive isotope of the impurity element under study [44]. The screened Coulomb potential of atomic rows and planes determines the anisotropic scattering of the particles emitted isotropically during decay. Along low-index crystal directions of single crystals, this anisotropic scattering results in well-defined channeling or blocking effects. Because these effects strongly depend on the initial position of the emitted particles, they result in emission patterns which are characteristic of the lattice site(s) occupied by the probe atoms. Several reviews on emission channeling can be found in the literature [44-47], and the technique has previously been used to investigate the lattice location of Fe [48], Co [49], $\mathrm{Mn}$ [49], and $\mathrm{Cu}[50]$ in $\mathrm{ZnO}$, in the low fluence $\left(\sim 10^{13} \mathrm{~cm}^{-2}\right)$ regime.

Each of the three samples implanted with stable ${ }^{56} \mathrm{Fe}$ for emission channeling experiments, were subsequently coimplanted with radioactive ${ }^{59} \mathrm{Fe}$ (with a half-life $t_{1 / 2}=46 \mathrm{~d}$ ) up to a fluence of $2 \times 10^{13} \mathrm{~cm}^{-2}$, by implanting the precursor isotope ${ }^{59} \mathrm{Mn}\left(t_{1 / 2}=4.6 \mathrm{~s}\right)$ which decays to ${ }^{59} \mathrm{Fe}$. The radioactive implantations were carried out at the on-line isotope separator facility ISOLDE at CERN, which provides mass-separated beams of radioactive $\mathrm{Mn}$ isotopes produced by means of $1.4-\mathrm{GeV}$ proton-induced nuclear fission from uranium carbide $\mathrm{UC}_{2}$ targets and chemically selective laser ion sources [51]. The $\beta^{-}$decay of ${ }^{59} \mathrm{Mn}$ transfers a recoil energy of about $200 \mathrm{eV}$ to its ${ }^{59} \mathrm{Fe}$ daughter. This ensures that the ${ }^{59} \mathrm{Fe}$ atoms are re-implanted, i.e. that they do not inherit the ${ }^{59} \mathrm{Mn}$ lattice site. The implantations were performed at room temperature, under a tilt angle of $7^{\circ}$ with respect to the surface normal, with an energy of $60 \mathrm{keV}$, resulting in a projected range $R_{\mathrm{P}}$ of $299 \AA$ and a $136 \AA$ straggling, estimated using the MARLOWE code [42]. Since the concentration of radioactive ${ }^{59} \mathrm{Fe}$ probes is at least two orders of magnitude below that of stable ${ }^{56} \mathrm{Fe}$, the increase in total Fe concentration compared to the samples used for RBS/C and SQUID measurements can be neglected. In addition, because the depth profiles of ${ }^{56} \mathrm{Fe}$ and ${ }^{59} \mathrm{Fe}$ overlap almost perfectly $\left(R_{\mathrm{p}}\right.$ is 297 and $299 \AA$ and straggling is 134 and $136 \AA$ for ${ }^{56} \mathrm{Fe}$ and ${ }^{59} \mathrm{Fe}$, respectively), one can assume that the ${ }^{59} \mathrm{Fe}$ probes accurately represent the site location behavior of all Fe impurities.

Angular-dependent emission yields of the $\beta^{-}$particles emitted during decay were measured at room temperature, along four crystallographic directions ([0001], [11102], [11101] and [2113]), in the as-implanted state and after in situ capless annealing in vacuum $\left(<10^{-5}\right.$ mbar $)$ up to $900^{\circ} \mathrm{C}$. These patterns were recorded using a position- and energy-sensitive detection system similar to that described in [52]. Using the many-beam formalism for electron channeling in single crystals [44], theoretical emission patterns were calculated for probes occupying substitutional $\mathrm{Zn}\left(S_{\mathrm{Zn}}\right)$ and $\mathrm{O}\left(S_{\mathrm{O}}\right)$ sites with varying root-mean-square (rms) displacements, the main interstitial sites and interstitial sites resulting from 
displacements along the [0001] or the basal directions. Quantitative lattice location is provided by fitting the experimental patterns with theoretical ones using the twodimensional fit procedure outlined in [52]. Corrections for secondary electrons that reach the detector were implemented by subtracting an isotropic background from every pattern. This secondary electron contribution was estimated based on Geant4 [53, 54] simulations of electron scattering, taking into account the elemental composition and geometry of the sample, sample-holder and vacuum chamber.

\section{Results and analysis}

Since the first question to address concerns the existence of room-temperature ferromagnetism, we start by discussing the SQUID magnetometry data at room temperature. This then sets the direction of the discussion in terms of structure (impurity lattice location and structural disorder) and a more detailed analysis of the magnetic behavior based on low-temperature SQUID magnetometry.

\subsection{SQUID magnetometry at room temperature}

For all samples, following some of the annealing steps, a small hysteresis could be resolved in the $300 \mathrm{~K} M-H$ data (magnetic moment $\mu$ as a function of applied field $H$ ) after subtracting the substrate's diamagnetic background (figures 2(a) and (b)). Figure 2(c) compiles the saturation moment $\mu_{\text {sat }}$ of this residual ferromagnetic component, for the three fluences, as a function of annealing temperature. The saturation moment remains consistently below $5 \times 10^{-7}$ emu and appears to increase with annealing temperature. We attribute this residual ferromagnetism to sample contamination, since there is no correlation with implanted fluence and since the same type of residual hystereses are observed in unimplanted control samples [2]. The apparent increase in saturation moment with annealing temperature (figure 2) is simply a consequence of the increase in number of processing steps, i.e. an increasing number of potentially contaminating events. In any case, one cannot exclude that some residual ferromagnetic moment arises from intrinsic ferromagnetism associated with the Fe doping or other beam-induced defects such as vacancies or self-interstitials. Based on the maximum saturation value for each fluence, table 1 lists the maximum ferromagnetic moment per $\mathrm{Fe}$ atom, assuming that all $\mathrm{Fe}$ atoms equally contribute to the ferromagnetic component. Ferromagnetic-like behavior can also be observed if a fraction of the implanted $\mathrm{Fe}$ precipitates in superparamagnetic $\alpha$-Fe clusters, as demonstrated by Zhou et al [9-12]. Table 1 also lists the maximum fraction of precipitated Fe, assuming that all the ferromagnetic-like moment originates from $\alpha$-Fe clusters with a moment per Fe atom of $2.2 \mu_{\mathrm{B}}$ (of bulk $\alpha$-Fe). Note that the higher fraction for the lower fluence sample is merely a consequence of dividing a similar saturation moment by a much smaller number of atoms. Since the fraction of $\mathrm{Fe}$ atoms in clusters is expected to increase with Fe concentration [11], one can conclude that the maximum fraction of clustered $\mathrm{Fe}$ in our samples is given by the value obtained for the highest fluence, i.e. less than $1 \%$.
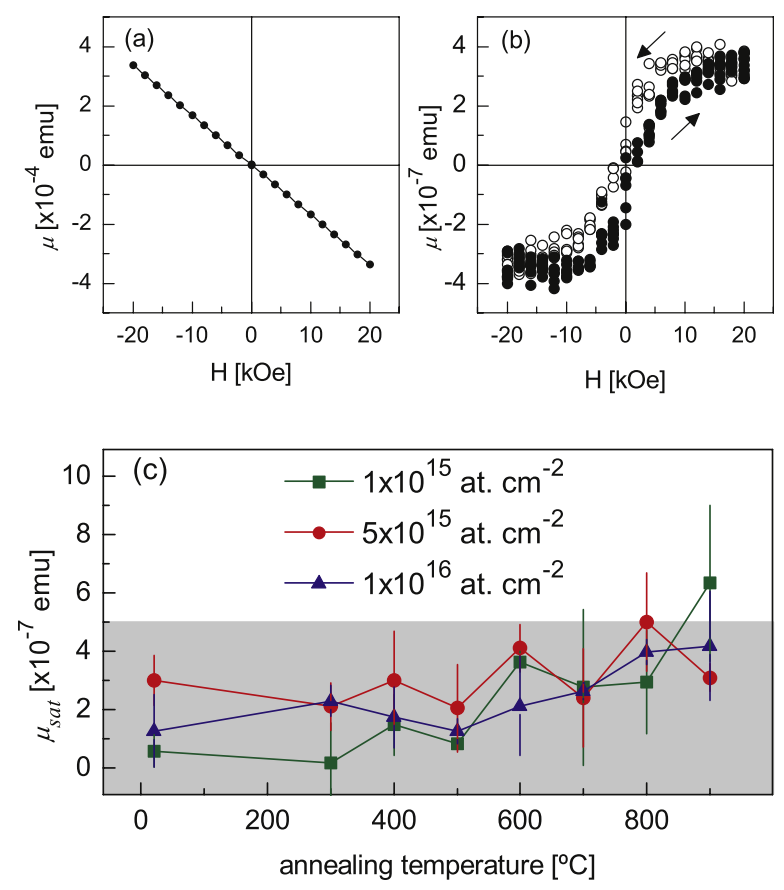

Figure 2. (a) $300 \mathrm{~K} M-H$ data of sample $\mathrm{S} 1$ following $600^{\circ} \mathrm{C}$ annealing. (b) Data after subtraction of the diamagnetic component estimated from the linear fit of the high-field (10-20 kOe) magnetization (arrows indicate increasing and decreasing field). (c) Saturation moment $\mu_{\text {sat }}$ for all three samples, as a function of annealing temperature, obtained from the linear fit to the $300 \mathrm{~K}$ $M-H$ data. All three samples have an area of approximately $0.25 \mathrm{~cm}^{2}$ and, therefore, the data can be compared directly. The shaded area below $5 \times 10^{-7}$ emu corresponds to the typical magnitude of the signal resulting from ferromagnetic-like contamination and measurement artifacts, i.e. the reliability limit for the detection of ferromagnetism $[2,6]$.

Table 1. Comparison between the amount of $\mathrm{Fe}$ atoms in each sample and the ferromagnetic saturation moment of the $300 \mathrm{~K} M-H$ data: maximum moment per $\mathrm{Fe}$ atom $\left(\mu_{\mathrm{Fe}}\right)$, assuming that all $\mathrm{Fe}$ atoms equally contribute to the ferromagnetic component; maximum fraction of $\mathrm{Fe}$ impurities in $\alpha$-Fe precipitates, assuming a moment per Fe atom of $2.2 \mu_{\mathrm{B}}$.

\begin{tabular}{|c|c|c|c|}
\hline Sample & $\begin{array}{l}\text { Fluence } \\
\left(\text { at. } \mathrm{cm}^{-2}\right)\end{array}$ & $\max . \mu_{\mathrm{Fe}}\left(\mu_{\mathrm{B}}\right)$ & $\begin{array}{l}\max . \alpha-\mathrm{Fe} \\
\text { fraction }(\%)\end{array}$ \\
\hline $\mathrm{S} 1$ & $1 \times 10^{15}$ & 0.18 & 8.1 \\
\hline S5 & $5 \times 10^{15}$ & 0.05 & 2.4 \\
\hline $\mathrm{S} 10$ & $1 \times 10^{16}$ & 0.02 & 0.8 \\
\hline
\end{tabular}

\subsection{Characterization of structural disorder using RBS/C}

Figure 3(a) shows representative RBS/C spectra measured in a backscattering geometry $\left(168^{\circ}\right)$ for an unimplanted sample and sample S5 after different annealing steps. As typical for $\mathrm{ZnO}$ (e.g. [55]), lattice disorder in the $\mathrm{Zn}$ sublattice accumulates in two regions: (1) in the bulk of the crystal (bulk peak), where the energy loss is maximum as the implanted ions are slowed down to the point that nuclear stopping dominates over electronic stopping and the host atoms are thus more efficiently displaced; (2) near the sample surface (surface peak), which acts as a sink for mobile defects created 

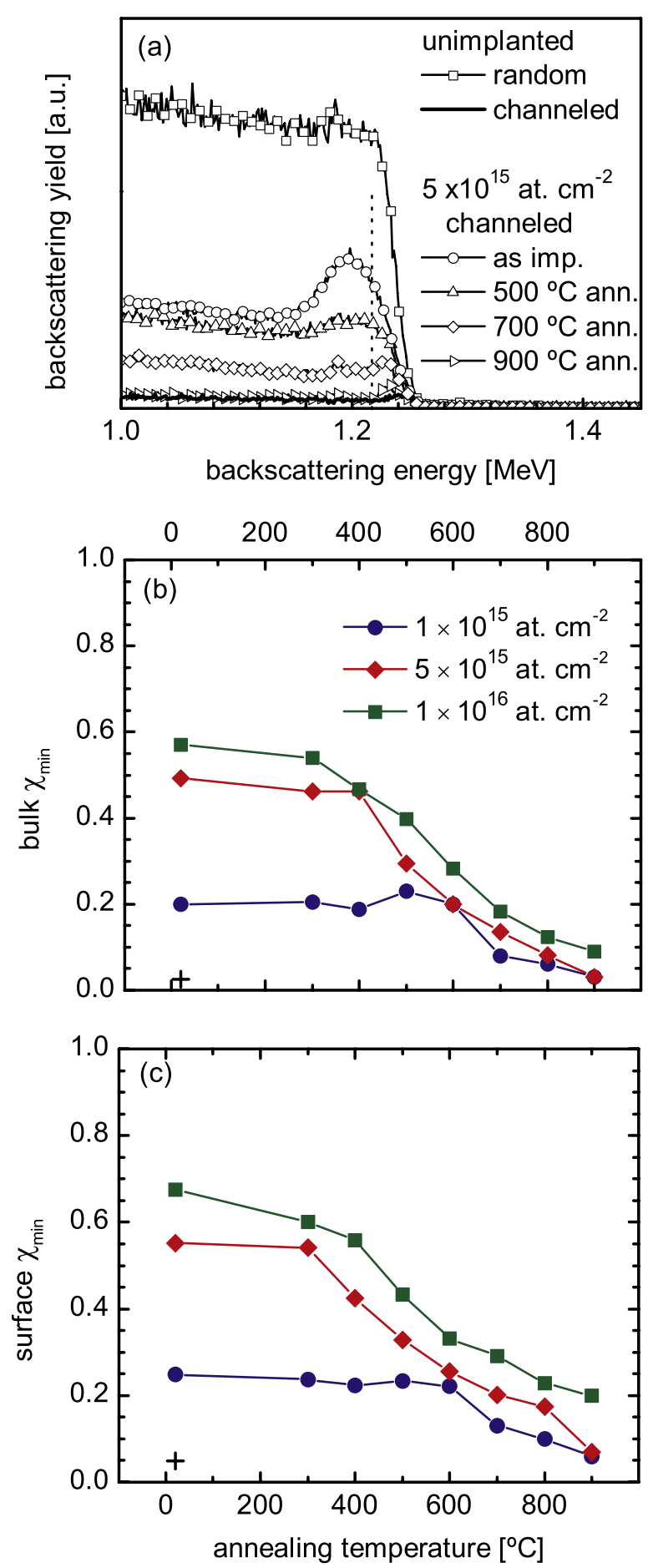

Figure 3. (a) RBS/C spectra measured in backscattering geometry $\left(168^{\circ}\right)$ aligned with the [0001] axis (channeling) for an unimplanted sample and sample S5 $\left(5 \times 10^{15} \mathrm{~cm}^{-2}\right)$ after different annealing steps, compared to the spectrum measured in a random beam orientation, for the unimplanted sample (the dashed line separates the surface and bulk energy windows used to calculate the corresponding $\chi_{\min }$ ). (b) Bulk and (c) surface $\chi_{\min }$ of the three samples, as a function of annealing temperature. Bulk and surface $\chi_{\min }$ of an unimplanted sample are also indicated $(+)$.

during the ion bombardment. The channeling minimum yield $\chi_{\min }$ is thus determined separately for these two regions. Bulk $\chi_{\min }$ are plotted in figure $3(\mathrm{~b})$ and surface $\chi_{\min }$ in figure $3(\mathrm{c})$, for the three fluences and annealing steps up to $900^{\circ} \mathrm{C}$. High fluences (of the order of $1 \times 10^{16} \mathrm{~cm}^{-2}$ ) are required to induce significant lattice disorder $\left(\chi_{\min }>50 \%\right)$, which reflects the efficient dynamic annealing which characterizes $\mathrm{ZnO}$ and is responsible for its known high radiation resistance.

The beam-induced damage is removed quite efficiently by thermal annealing. Both bulk and surface $\chi_{\text {min }}$ decrease with increasing annealing temperature, particularly between 400 and $900{ }^{\circ} \mathrm{C}$. After annealing at $900^{\circ} \mathrm{C}$, the bulk $\chi_{\text {min }}$ for fluences of $1 \times 10^{15}$ and $5 \times 10^{15} \mathrm{~cm}^{-2}$ (3\% in both cases) are nearly the same as prior to implantation $(2.5 \%)$. The recovery at the surface is also very efficient for these fluences, with $\chi_{\min }$ of $6 \%$ and $7 \%$ for $1 \times 10^{15} \mathrm{~cm}^{-2}$ and $5 \times 10^{15} \mathrm{~cm}^{-2}$, respectively, compared to $5 \%$ prior to implantation. For a fluence of $1 \times 10^{16} \mathrm{~cm}^{-2}$ the recovery is somewhat less efficient, indicating that higher fluence implantation creates defect complexes which are more stable with respect to thermal annealing. This is in agreement with the general rule of thumb that annealing of extended defects in semiconductors requires a temperature of about two thirds of the material's melting point (in units of K) [56], which for $\mathrm{ZnO}$ corresponds to about $1200^{\circ} \mathrm{C}$. Nevertheless, a variation of the $\chi_{\min }$ in the bulk region (i.e. in the region where the majority of the $\mathrm{Fe}$ impurities are located) from $67 \%$ in the as-implanted state down to $9 \%$ after $900{ }^{\circ} \mathrm{C}$ annealing is quite satisfactory for the purpose of this work. This brings up the question of how exactly the lattice recovery evolves with annealing within the bulk region. Figure 4 shows the RBS/C spectra measured in glancing geometry (which provides an increased depth resolution) of sample S10 after each annealing step. It shows that up to the $500^{\circ} \mathrm{C}$ annealing step, the bulk peak does not decrease uniformly. Instead, the decrease in backscattering yield is less pronounced in the $\mathrm{Fe}$ end of range (EOR) region than in the region between the EOR and the surface, which results in an apparent shift of the bulk peak to the EOR. This indicates that the damage in the EOR region is more stable, being annealed only at $700{ }^{\circ} \mathrm{C}$ and above, which in turn suggests that the defects responsible for the increased backscattering yield in the bulk peak form complexes with the Fe impurities. This is discussed in more detail below, together with the emission channeling results on the lattice location of the Fe impurities.

As a final remark on figure 4 , it is interesting to note that the low-temperature annealing (up to $500^{\circ} \mathrm{C}$ ) reveals an additional defect peak in the region between the surface and bulk defect peaks, which was also observed in Au-implanted $\mathrm{ZnO}$ [55] and coined as middle defect peak (MDP). In [55], the MDP was attributed to a localized band of lattice defects, which nucleates in the near surface region due to incomplete dynamic annealing during ion bombardment.

\subsection{Impurity lattice site location using $\beta^{-}$emission channeling}

Figures 5(a)-(d) show the experimental emission patterns

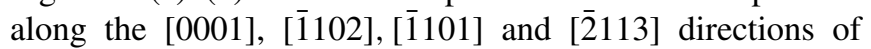
sample S1 following $300^{\circ} \mathrm{C}$ annealing. Figures 5(e)-(h) show the best fits of the corresponding theoretical yields, obtained 


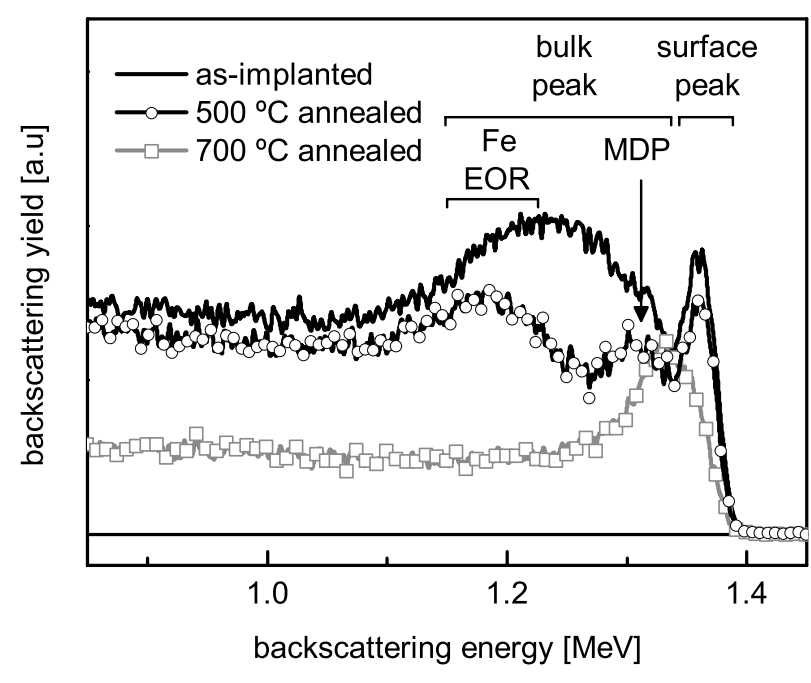

Figure 4. RBS/C spectra measured in glancing geometry $\left(15^{\circ}\right.$ exit angle with respect to the sample surface) in the channeling orientation of sample $\mathrm{S} 10\left(1 \times 10^{16} \mathrm{~cm}^{-2}\right)$ for the different annealing stages. Four different damage regions are indicated: surface peak, bulk peak and, within the bulk peak, the region corresponding to the Fe end of range (EOR) and the middle defect peak (MDP).

by varying the fraction of Fe on substitutional $S_{\mathrm{Zn}}$ sites (best fit for a fraction of $87(5) \%$ ) and varying rms displacement $u_{1}$ from the ideal $S_{\mathrm{Zn}}$ site (best fit for $u_{1}=0.08(1) \AA$ ). The remaining $\mathrm{Fe}$ fraction contributes with an isotropic emission yield, and is discussed below. Figure 6 compiles the fit results for the different fluences and annealing stages: the fractions of Fe impurities in $S_{\mathrm{Zn}}$ are plotted in figure 6(a) and the corresponding rms displacements $u_{1}$ in figure 6(b).

3.3.1. Random fraction. The random fractions are virtually independent of annealing temperature and increase with implanted fluence. They correspond to Fe atoms which are located either in (1) crystalline secondary phases which are not coherent with the host structure, or in (2) highly disordered regions. Based on the work of Zhou et al, case (1) could correspond to metallic $\alpha$-Fe nanoclusters. However, these can be excluded based on the SQUID measurements at room temperature, from which we concluded that the maximum Fe fractions in $\alpha$-Fe nanoclusters was below $1 \%$ (see above). We interpret the observed random fraction as case (2), i.e. Fe impurities located in highly disordered (or even amorphous) regions. Because the random $\mathrm{Fe}$ fractions are very small and do not induce measurable ferromagnetism, their (potentially) magnetic behavior is assumed in the remainder of this paper to be negligible compared to the net magnetic moment of the substitutional Fe fraction. Note that the random fractions may even be somewhat overestimated, as a result of electron dechanneling due to beam-induced lattice disorder [44-47].

3.3.2. Substitutional Fe. The substitutional fraction consists of $\mathrm{Fe}$ atoms occupying sites which are epitaxially aligned with the $\mathrm{Zn}$ sublattice along the four measured directions. These sites can either be (1) true $\mathrm{Zn}$ sites in the $\mathrm{ZnO}$



Figure 5. (a)-(d) Normalized experimental emission patterns along the [0001], [1102], [11101] and [2113] directions of sample S1 $\left(1 \times 10^{15} \mathrm{~cm}^{-2}\right)$ following $300^{\circ} \mathrm{C}$ annealing. (e)-(h) Corresponding best fits of theoretical yields, obtained by varying the fraction of $\mathrm{Fe}$ on substitutional $S_{\mathrm{Zn}}$ sites (best fit for a fraction of 77(5)\%) and varying its rms displacement $u_{1}$ from the ideal $S_{\mathrm{Zn}}$ site (best fit for $\left.u_{1}=0.08(1) \AA\right)$.

wurtzite structure or (2) Fe sites in a secondary phase crystallite which are aligned with the $\mathrm{Zn}$ sublattice of the host $\mathrm{ZnO}$ structure. Even though the crystallites of the spinel ferrite $\mathrm{ZnFe}_{2} \mathrm{O}_{4}$ identified by Zhou et al $[10,11]$ can be crystallographically aligned with the $\mathrm{ZnO}$ wurtzite structure, the Fe sublattice in such structures is not coherent with the $\mathrm{Zn}$ sublattice of the host $\mathrm{ZnO}$ matrix. Hence, we are left with case (1). However, occupying nearly ideal $\mathrm{Zn}$ sites in the $\mathrm{ZnO}$ wurtzite structure does not necessarily mean that the $\mathrm{Fe}$ is uniformly distributed. From the EC results, one can only conclude that the majority of the $\mathrm{Fe}$ impurities are located in the cation sites of a wurtzite alloy of formula $\mathrm{Zn}_{1-x} \mathrm{Fe}_{x} \mathrm{O} ; x$ may vary locally creating Fe-rich and $\mathrm{Fe}$-poor regions. Such aggregation effects are limited by the mobility of the Fe impurities; although randomly incorporated in the crystal during implantation, they may become mobile and aggregate at sufficiently high annealing temperatures. Because aggregation implies a decrease of the average minimum distance between neighboring $\mathrm{Fe}$ impurities, it enhances the magnetic interactions between the Fe localized moments. However, emission channeling is unable to probe the uniformity of the Fe distribution. Aggregation is discussed in detail next, based on the magnetometry measurements. 


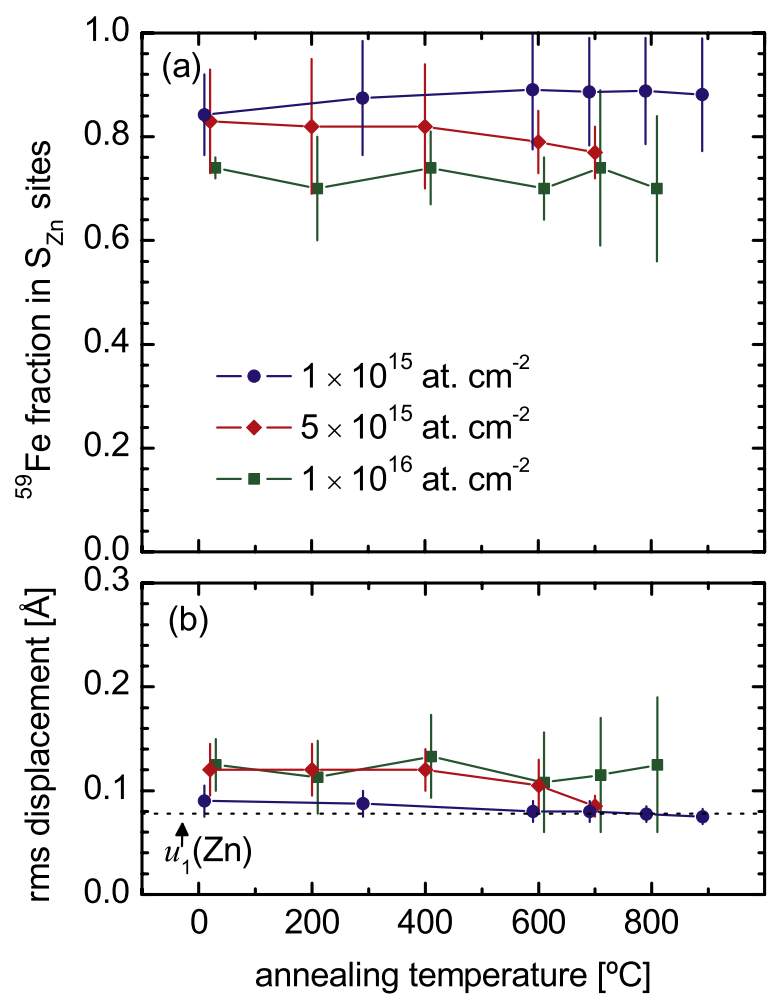

Figure 6. (a) Fractions of ${ }^{59} \mathrm{Fe}$ impurities in $\mathrm{Zn}$-substitutional $\left(S_{\mathrm{Zn}}\right)$ sites and (b) the corresponding rms displacements $u_{1}$ obtained from fitting the experimental patterns with theoretical ones, as described in the text (the dashed line indicates the thermal vibration amplitude of the $\mathrm{Zn}$ atoms in the lattice).

However, before analyzing the magnetism in more detail, it is worth discussing the fluence and temperature dependence of $u_{1}$. The rms displacement $u_{1}$, obtained from fitting the EC patterns, can in principle be divided into two components: the thermal vibration amplitude of Fe impurities in $\mathrm{Zn}$ sites, which is expected to be similar to that of the $\mathrm{Zn}$ atoms $\left(u_{1}(\mathrm{Zn})=0.08 \AA\right)$ [57], and static displacements from the ideal $\mathrm{Zn}$ sites. The fitted $u_{1}$ values, plotted in figure $6(\mathrm{~b})$, show that the degree of displacement from ideal $\mathrm{Zn}$ sites, and thus the disorder in the vicinity of the Fe impurities, increases with fluence, as expected and observed in the RBS/C data. More importantly, it shows that the disorder in the close vicinity of the Fe impurities anneals only between 600 and $800^{\circ} \mathrm{C}$, as $u_{1}$ decreases to the thermal vibration amplitude, which is consistent with our previous emission channeling experiments on very low fluence Fe-implanted $\mathrm{ZnO}$ [48]. Combined with the RBS/C results above, this provides a quite detailed insight into the overall damage accumulation and annealing in the EOR region. Thermal annealing up to $600{ }^{\circ} \mathrm{C}$ efficiently anneals the damage in the region between the Fe EOR and the surface, as shown by the RBS/C data in figure 4. However, most of the damage in the EOR region persists, most likely because it is stabilized by impurity-defect complexes involving the Fe impurities and native point defects created during implantation, slightly displacing the Fe atoms from the ideal $\mathrm{Zn}$ sites and thus explaining the $u_{1}$ values lying significantly above the thermal vibration amplitude of $\mathrm{Zn}$ in $\mathrm{ZnO}$. According to the extensive work of Weyer et al using Mössbauer spectroscopy [58-60], these impurity-defect complexes are most likely Zn-vacancy related, which is supported by EPR measurements [61]. Our data suggest that these impurity-defect complexes dissociate upon annealing between 600 and $800^{\circ} \mathrm{C}$ : as the complexes dissociate, the lattice defects ( $\mathrm{Zn}$ sublattice related) are able to diffuse away from the Fe impurities. This is consistent with the RBS/C data in figure 4, where the $\mathrm{Zn}$-sublattice disorder in the EOR region appears to diffuse towards the surface upon annealing at $T>500{ }^{\circ} \mathrm{C}$, as discussed above.

\subsection{Low-temperature magnetization and correlation with the structural properties}

Let us start by summarizing the main findings so far:

(i) The EC data show that the large majority of Fe impurities occupy $\mathrm{Zn}$ sites in the $\mathrm{ZnO}$ wurtzite structure, i.e. the implanted layer consists of a single-crystalline wurtzite alloy $\mathrm{Zn}_{1-x} \mathrm{Fe}_{x} \mathrm{O}$, although $\mathrm{Fe}$ aggregation into Fe-rich regions cannot be excluded.

(ii) The absence of measurable room-temperature ferromagnetism (or superparamagnetism) shows that the small fraction of non-substitutional $\mathrm{Fe}$, i.e. the random fraction in the EC analysis, cannot be attributed to known secondary phases in Fe-implanted $\mathrm{ZnO}$, i.e. metallic $\alpha$-Fe or the spinel ferrite $\mathrm{ZnFe}_{2} \mathrm{O}_{4}$.

(iii) The RBS/C data show that thermal annealing decreases the degree of disorder of the $\mathrm{Zn}_{1-x} \mathrm{Fe}_{x} \mathrm{O}$ layer from a dense damage profile to a nearly fully recovered crystal structure. Combining the RBS/C and the EC results, we conclude that point-like native defects created by implantation form impurity-defect complexes with the Fe impurities. Thermal annealing at $600{ }^{\circ} \mathrm{C}$ and above induces the dissociation of these complexes, allowing the native defects to diffuse towards the surface.

(iv) The single-crystalline wurtzite alloy $\mathrm{Zn}_{1-x} \mathrm{Fe}_{x} \mathrm{O}$ does not display room-temperature ferromagnetism in a wide range of defect density.

Two major questions remain unanswered:

(i) If not high-temperature ferromagnetism, what is the magnetic behavior of wurtzite $\mathrm{Zn}_{1-x} \mathrm{Fe}_{x} \mathrm{O}$ and how does it depend on the density of lattice defects?

(ii) How does thermal annealing affect the uniformity of $x$ across the $\mathrm{Zn}_{1-x} \mathrm{Fe}_{x} \mathrm{O}$ layer and, in turn, how does that affect the magnetic interaction between substitutional $\mathrm{Fe}$ impurities?

Experimentally addressing question (2) is extremely challenging. We show below that addressing question (1) allows us to infer an answer to question (2). In order to answer (1), we will focus on sample S5 $\left(5 \times 10^{15} \mathrm{~cm}^{-2}\right)$ for the following reasons. As we show next, the large number of paramagnetic impurities in the $\mathrm{ZnO}$ substrates (relative to the number of implanted $\mathrm{Fe}$ ions) dominates the magnetic signal of sample $\mathrm{S} 1\left(1 \times 10^{15} \mathrm{~cm}^{-2}\right)$, thus precluding a detailed analysis. On the other hand, due to the lower quality of 

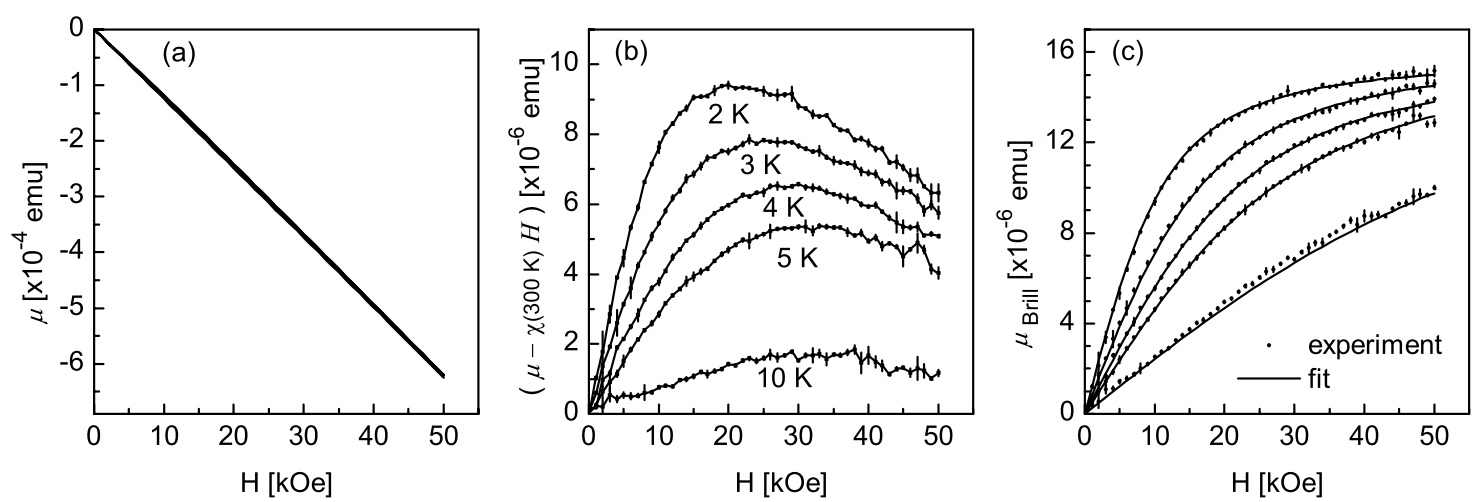

Figure 7. (a) Isothermal $M-H$ data of sample $S 5$ prior to implantation at 2, 3, 4, 5, 10 and $300 \mathrm{~K}$ (data appear to overlap), measured with decreasing field (from 50 to $0 \mathrm{kOe}$ ) parallel to the sample plane. (b) Data after subtraction of the linear component (estimated from the linear fit of the $300 \mathrm{~K}$ data in the 20-50 kOe range). (c) Brillouin-like component and corresponding fit, according to the model described in the text.

the EC data of sample S10 $\left(1 \times 10^{16} \mathrm{~cm}^{-2}\right)$, it cannot be determined beyond doubt if the damage in the vicinity of the Fe impurities is completely annealed, making sample S10 less suitable for investigating possible changes in magnetic behavior upon dissociation of the impurity-defect complexes. In addition, if the $\mathrm{Fe}$ impurities do aggregate, this should occur at lower annealing temperatures in S10 due to the higher Fe concentration, which makes it more difficult to separate the effects of defect annealing and Fe aggregation on the magnetometry data.

In the following, we analyze the magnetometry data of sample S5 at low temperatures, focusing on the relevant processing stages: prior to implantation, in order to characterize the magnetic background of the $\mathrm{ZnO}$ substrate; as-implanted, when the uniformity of the $\mathrm{Fe}$ distribution should be maximum; after $500^{\circ} \mathrm{C}$ annealing, when most of the damage which does not overlap with the Fe profile has been removed; and after $700^{\circ} \mathrm{C}$ annealing, when most of the defects which more directly interact with the Fe impurities have been removed.

\subsubsection{As-grown $\mathrm{ZnO}$ substrate. Before analyzing the} data for the implanted sample, it is necessary to carefully characterize the substrate background. Figure 7(a) shows the isothermal $M-H$ data of sample S5 prior to implantation at several temperatures from 2 to $300 \mathrm{~K}$ (all data appear to overlap). The nonlinear component of the $M-H$ curves at low temperatures is revealed (figure 7(b)) after subtracting the (diamagnetic) linear component at $300 \mathrm{~K}$ estimated from the linear fit of the high-field range (20-50 kOe). The shape of these curves resembles a paramagnetic component (following the Brillouin function) superimposed on a small negative slope. The Brillouin-like component can be attributed to paramagnetic impurities, most likely transition metal impurities in the ppm range incorporated during hydrothermal growth [62]. The negative slope at low temperature can be explained by a decreasing van Vleck paramagnetic susceptibility of the $\mathrm{ZnO}$ substrate with decreasing temperature (from 300 to $\leq 10 \mathrm{~K}$ ), due to the temperature dependence of the $\mathrm{ZnO}$ bandgap $E_{\mathrm{g}}$. This has been observed in a number of semiconductor materials and is discussed in some detail in [63] for GaAs substrates. The low-temperature data can therefore be fitted using the ansatz

$$
\mu(H, T)=\mu_{\text {Brill }}(H, T)+\Delta \mu_{\mathrm{vV}}^{\mathrm{ZnO}}(H, T),
$$

where $\mu_{\text {Brill }}$ is the Brillouin-like magnetic moment of the paramagnetic impurities and $\Delta \mu_{\mathrm{vV}}^{\mathrm{ZnO}}$ accounts for the variation of the van Vleck paramagnetic susceptibility of the $\mathrm{ZnO}$ substrate. The Brillouin-like component can be expressed as

$$
\mu_{\mathrm{Brill}}(H, T)=N g J \mu_{\mathrm{B}} B_{J}(x), \quad\left(x \equiv \frac{g J \mu_{\mathrm{B}} H}{k_{\mathrm{B}} T}\right),
$$

where $N$ is the number of paramagnetic impurities, $g$ is the Landé factor, $J$ is the total angular momentum quantum number and $B_{J}(x)$ is the Brillouin function:

$$
B_{J}(x)=\frac{2 J+1}{2 J} \operatorname{coth}\left(\frac{2 J+1}{2 J} x\right)-\frac{1}{2 J} \operatorname{coth}\left(\frac{1}{2 J} x\right) .
$$

Assuming that the orbital momentum $L$ is fully quenched $(L=0)$, which we show below to be a good approximation in this case, $J$ is given by the spin quantum number $S(J=S)$ and $g=2.00$. To a first approximation, we can assume that the van Vleck paramagnetic susceptibility varies very little from 10 to $2 \mathrm{~K}$, so that $\Delta \mu_{\mathrm{vV}}$ can be taken as

$$
\Delta \mu_{\mathrm{vV}}^{\mathrm{ZnO}}(H, T)=\Delta \chi_{\mathrm{vV}}^{\mathrm{ZnO}} H,
$$

where $\Delta \chi_{\mathrm{vV}}^{\mathrm{ZnO}}$ is independent of temperature and field. Figure 7(c) shows the data in (b) and the fit using the described ansatz, with $S, N$ and $\Delta \chi_{\mathrm{vV}}^{\mathrm{ZnO}}$ as free parameters. The fit reproduces the data quite well, with $S=2.6(2), N=$ 3.11(3) (i.e. a concentration of $4 \times 10^{16} \mathrm{~cm}^{-3}$ ) and $\Delta \chi_{\mathrm{vV}}^{\mathrm{ZnO}}$ corresponding to about $1 \%$ of the diamagnetic susceptibility at $300 \mathrm{~K}$. The Brillouin component is consistent with $\mathrm{Fe}^{3+}$ $\left(\mathrm{d}^{5} S=5 / 2\right.$ ), a common impurity in hydrothermally grown $\mathrm{ZnO}$ [64], particularly in the substrates used here (grown by CrysTec $\mathrm{GmbH}$ ) [62]. The small $\Delta \chi_{\mathrm{vV}}^{\mathrm{ZnO}}$ is consistent with the expected small temperature dependence of the van Vleck paramagnetic susceptibility. Note that all the data are fitted simultaneously, i.e. the same $N, S$ and $\Delta \chi_{\mathrm{VV}}^{\mathrm{ZnO}}$ values are optimized simultaneously for the different temperatures.

The derivation of the Brillouin function does not take into account anisotropic crystal fields. In wurtzite materials 
such as $\mathrm{ZnO}$, substitutional impurities are subject to a trigonal crystal field. Via the spin-orbit interaction, this anisotropic crystal field induces a strong magnetic anisotropy, which can be described by a zero-field splitting of the fundamental state and by an anisotropic effective $g$-factor in an effective spin Hamiltonian $H_{\mathrm{s}}$ of the form

$$
H_{\mathrm{s}}=\mu_{\mathrm{B}} g_{\|} H_{z} S_{z}+\mu_{\mathrm{B}} g_{\perp}\left(H_{x} S_{x}+H_{y} S_{y}\right)+D S_{z}^{2},
$$

where $S$ is the spin quantum number, $D$ describes the zero-field splitting, and $g_{\|}$and $g_{\perp}$ are the effective $g$-factors for directions of magnetic field parallel and perpendicular to the wurtzite $c$-axis, respectively. Therefore, in general, the $\mu(H, T)$ behavior of paramagnetic impurities in the crystal field of the $\mathrm{ZnO}$ wurtzite lattice cannot be correctly reproduced by the Brillouin function. The fact that it does in our case shows that the zero-field splitting constant for this particular impurity in $\mathrm{ZnO}$ is very small. This is typically the case for $S=5 / 2$ moments (e.g. $D=-0.0074 \mathrm{meV}$ for $\mathrm{Fe}^{3+} \mathrm{d}^{5}$ in $\mathrm{ZnO}$ [65], $D=-0.0027 \mathrm{meV}$ for $\mathrm{Mn}^{2+} \mathrm{d}^{5}$ in $\mathrm{ZnO}$ [66] and $D=-0.0093 \mathrm{meV}$ for $\mathrm{Mn}^{2+} \mathrm{d}^{5}$ in $\mathrm{GaN}$ [67]). In fact, even for $\mathrm{Co}^{2+}\left(\mathrm{d}^{7}, S=3 / 2\right)$, where $D$ is significantly larger $(0.342 \mathrm{meV})$, the in-plane magnetization can be reasonably well described by the Brillouin function [25].

Because the implantation affects only a very thin layer of less than $1 / 1000$ of the whole substrate thickness, this substrate background can be taken as unchanged after implantation, allowing us to isolate the magnetization associated with the implanted $\mathrm{Fe}$ impurities and beaminduced defects.

\subsubsection{After implantation at room temperature. Figure 8(a)} shows the low-temperature $M-H$ curves of sample S5 in the as-implanted state, after subtraction of the diamagnetic component estimated from the linear fit of the high-field (10-20 kOe) magnetization at $300 \mathrm{~K}$. Fitting the data with the same ansatz (1) gives a reasonable agreement (figure 8(b)). To be more precise, the data are fitted to a sum of two terms of the form of (1): for one of them, the parameters $N, S$ and $\Delta \chi_{\mathrm{vV}}^{\mathrm{ZnO}}$ are set (i.e. fixed) to those obtained prior to implantation (i.e. due to the contaminant paramagnetic background), so that the free $N, S$ and $\Delta \chi_{\mathrm{vV}}^{\mathrm{ZnFeO}}$ parameters of the second term describe the implanted layer. The agreement can be further improved by allowing $\Delta \chi_{\mathrm{vV}}^{\mathrm{ZnFeO}}$ to be temperature dependent, which is implemented in the fitting as follows. First, the $2 \mathrm{~K}$ $M-H$ curve, which carries more 'information' as it provides a wider range of $H / T$ (and thus of $x$ in equation (2)), is fitted with $N, S$ and $\Delta \chi_{\mathrm{vV}}^{\mathrm{ZnFeO}}$ as free parameters. The $M-H$ curves for 5 and $10 \mathrm{~K}$ are then fitted separately, fixing the $N$ and $S$ values determined from the $2 \mathrm{~K} M-H$, and varying only $\Delta \chi_{\mathrm{vV}}^{\mathrm{ZnFeO}}$. Figure $8(\mathrm{~b})$ compares the experimental data to the best fit, after subtraction of $\Delta \chi_{\mathrm{vV}}^{\mathrm{ZnFeO}} H$ in order to more clearly show the Brillouin component. The agreement is again very good, supporting the existence of two types of paramagnetism: Brillouin-like and van Vleck-like.

- Brillouin-like component. The best fit is obtained for $S=2.5(1)$, i.e. $S=5 / 2$ within the error, which is the expected behavior for the implanted $\mathrm{Fe}$ if it is incorporated

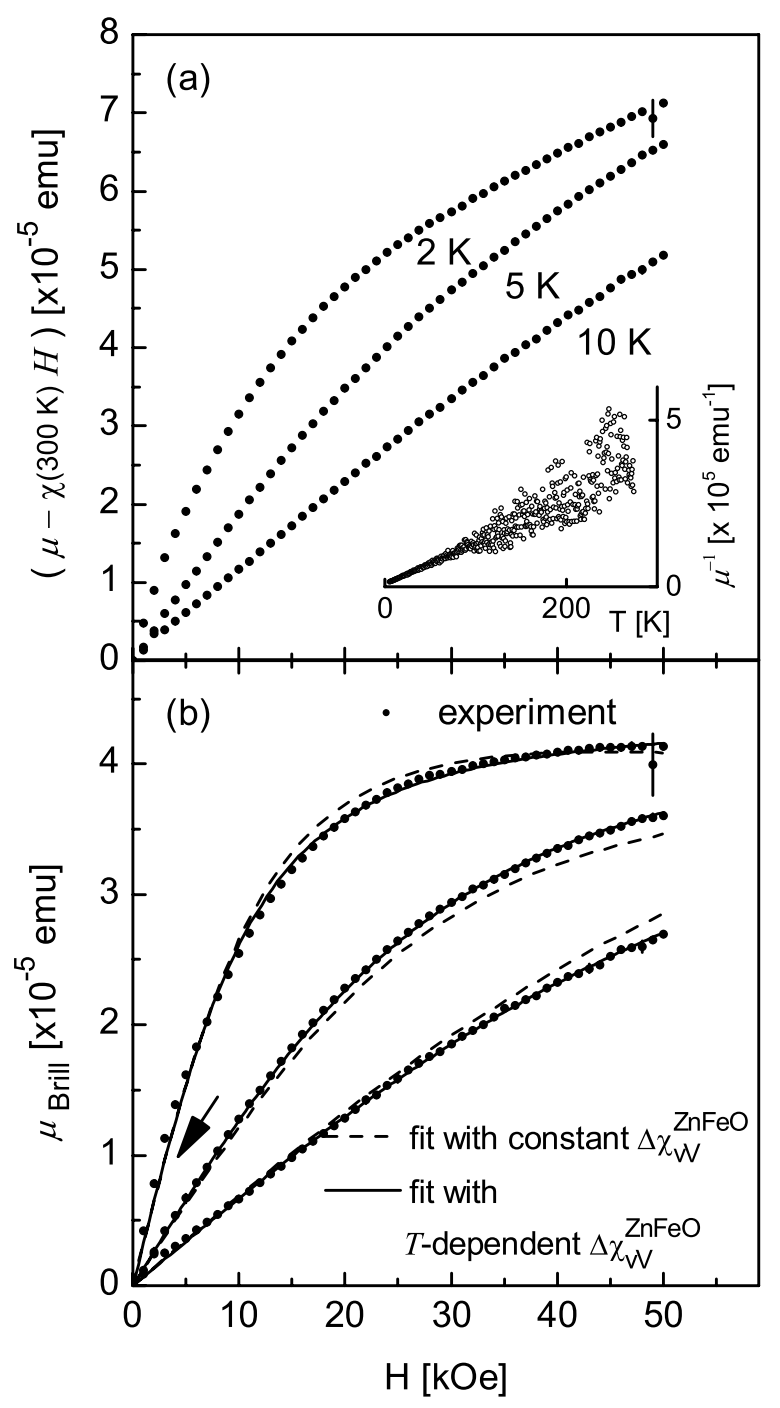

Figure 8. (a) Isothermal $M-H$ data of sample $\mathrm{S} 5$ in the as-implanted state, at 2, 5 and $10 \mathrm{~K}$, corrected for the diamagnetic susceptibility at $300 \mathrm{~K}$ (inset: $M-T$ data of the same sample, with an applied field of $50 \mathrm{kOe}$, corrected of the diamagnetic component following the procedure described in [34], and plotted as $\mu^{-1}(T)$ in order to show the paramagnetic Curie behavior). (b) Brillouin-like component of the experimental data and corresponding theoretical fits according to the model described in the text. The best fit is obtained for $S=5 / 2$, with $\Delta \chi_{\mathrm{vV}}^{\mathrm{ZnFeO}}$ taken as temperature independent (dashed line) or temperature dependent (solid line). The arrow indicates the region of poor fit even when $\Delta \chi_{\mathrm{vV}}^{\mathrm{ZnFO}}$ is allowed to vary.

as $\mathrm{Fe}^{3+} \mathrm{d}^{5}$. The zero-field splitting $D$ for $\mathrm{Fe}^{3+} \mathrm{d}^{5}$ is sufficiently small $(-0.0074 \mathrm{meV})$ [65] for the Brillouin function to be a good approximation. Although $2+$ is the 'neutral' charge state of Fe impurities substituting group-II $\mathrm{Zn}$ in $\mathrm{ZnO}$, a $3+$ charge state may result from charge transfer from neighboring acceptor defects. It has been suggested, based on Mössbauer experiments, that $\mathrm{Fe}$ impurities in impurity-defect complexes with $\mathrm{Zn}$ vacancies (acceptors) created during $\mathrm{Fe}$ implantation are indeed in the 3+ charge state [58-60]. This is also supported by EPR measurements [61], and is consistent with our RBS/C and EC results discussed above, which also indicate the 
formation of such complexes. The perturbation induced by the neighboring native defects on the crystal field is extremely small (of the order of $10^{-6} \mathrm{meV}$ [61]) and, therefore, the Brillouin function remains a good approximation.

The small deviations from the Brillouin fit (arrow in figure $8(\mathrm{~b})$ ) can be attributed to a small fraction of the implanted $\mathrm{Fe}$ impurities in the $2+$ charge state. In principle one could attempt to incorporate such a fraction in the fit. However, $D, g_{\|}$and $g_{\perp}$ (equation (5)) are unknown for $\mathrm{Fe}^{2+}$ in $\mathrm{ZnO}$, which would require too many free parameters in the fit. For $\mathrm{Mn}^{3+}$ in $\mathrm{GaN}$, in principle a very similar case $(S=2), D=0.27 \mathrm{meV}$ [68], i.e. sufficiently high to induce the observed deviations from Brillouin behavior in figure 8(b).

Another important observation concerns the fitted number of paramagnetic centers $N=5.6(4) \times 10^{14}$, i.e. only about $45 \%$ of the number of implanted $\mathrm{Fe}$ impurities. The decreased $N$ can in part be attributed to fitting the data with a high spin $\mathrm{Fe}^{3+} \mathrm{d}^{5}(S=5 / 2)$, when a fraction may in fact be $\mathrm{Fe}^{2+} \mathrm{d}^{6}(S=2)$. In addition, it is possible that the magnetic moment of $\mathrm{Fe}$ impurities in highly damaged regions (the random fraction in EC, i.e. $16 \%$ of the implanted $\mathrm{Fe}$ ) may be quenched. However, even these two effects combined are insufficient to explain the 'missing' $55 \%$ of the Fe impurities. This suggests an additional source of Fe moment quenching: the mutual compensation of the magnetic moment of neighboring $\mathrm{Fe}$ impurities due to the antiparallel alignment of their spins, imposed by strong nearest-cation-neighbor antiferromagnetic interactions. Even if the Fe impurities are randomly incorporated in $\mathrm{Zn}$ sites in $\mathrm{ZnO}$ upon implantation, there is a finite fraction that occupies nearest-cation-neighbor sites: $34 \%$ according to the Behringer equation [69] taking with $x=x_{\mathrm{p}}$. If the magnetic interaction between neighboring $\mathrm{Fe}$ moments is antiferromagnetic, the resulting antiparallel alignment of their spins renders this fraction 'invisible' in the magnetization data. A similar moment-compensation effect has been observed in other DMS systems, e.g. Co-doped $\mathrm{ZnO}$ [27, 28] and Cr-doped GaN [34].

- van Vleck-like component. There is an important observation, independent of any fitting, which we have not mentioned so far: the total magnetic moment at $2 \mathrm{~K}$ and $50 \mathrm{kOe}$ (figure $8(\mathrm{a})$ ) of $7.1 \times 10^{-5} \mathrm{emu}$, is larger than that expected from the Fe impurities even if all were to contribute with the maximum spin-only value of $5 \mu_{\mathrm{B}}$ $\left(5.8 \times 10^{-5}\right.$ emu in total $)$, even more so, as the curve seems to be far from saturation. There is then an additional contribution from paramagnetic defects in the implanted layer other than the Fe impurities. The fitting presented above indicates that this additional contribution can be described by a van Vleck-like paramagnetic susceptibility of the form

$$
\begin{aligned}
\chi_{\mathrm{vV}}^{\mathrm{ZnFeO}}(T) & =\frac{\mu_{\mathrm{vV}}^{\mathrm{ZnFeO}}(H, T)}{H} \\
& =\chi_{\mathrm{vV}}^{\mathrm{ZnFeO}}(300 \mathrm{~K})+\Delta \chi_{\mathrm{vV}}^{\mathrm{ZnFeO}}(T)
\end{aligned}
$$

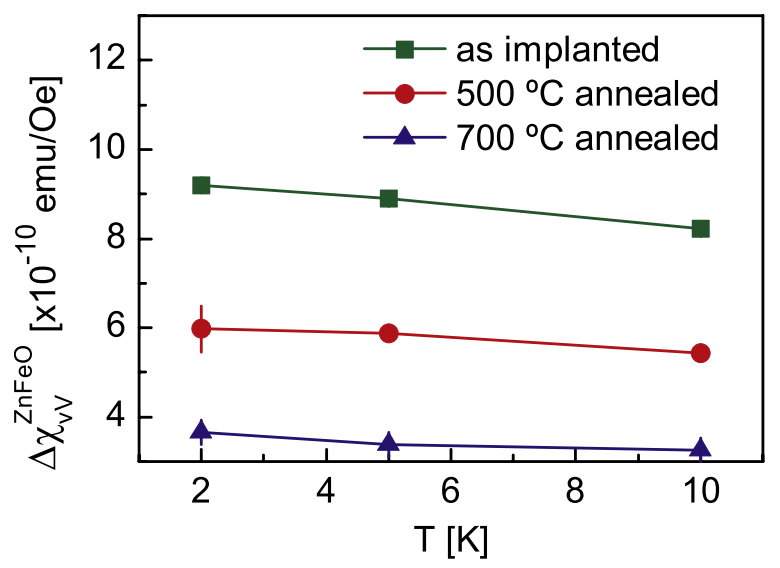

Figure 9. Measurement temperature and annealing temperature dependence of $\Delta \chi_{\mathrm{vV}}$ (equation (4)), obtained from fitting the low-temperature $M-H$ data of sample S5.

We attribute this van Vleck-like paramagnetic component to native defects created during implantation, which is further supported as we analyze the changes induced by annealing, below. The temperature dependence of $\Delta \chi_{\mathrm{vV}}^{\mathrm{ZnFeO}}$ is shown in figure 9. This van Vlecklike susceptibility component decreases with increasing temperature, which is the opposite to what was observed above for the van Vleck susceptibility of the substrate $\Delta \chi_{\mathrm{vV}}^{\mathrm{ZnO}}$ (due to the temperature dependence of the bandgap), and therefore supports their distinct origins $\left(\Delta \chi_{\mathrm{vV}}^{\mathrm{ZnO}}\right.$ from the perfect crystal, and $\Delta \chi_{\mathrm{vV}}^{\mathrm{ZnFeO}}$ from the beam-induced damage). It is, however, very difficult to estimate the absolute magnitude of either van Vleck-like susceptibilities, since it is virtually impossible to separate it from the purely (Langevin-like) diamagnetic susceptibility solely based on our data. Moreover, it is possible that part of the fitted $\Delta \chi_{\mathrm{vV}}^{\mathrm{ZnFO}}$ component originates, in fact, from the antiferromagnetically coupled Fe impurities, contributing with a non-vanishing (antiferro)magnetic susceptibility. In any case, as discussed above, a source of van Vleck-like magnetization must exist, since the Fe impurities alone cannot account for the magnitude of the observed magnetization. However, the detailed description of such defect-related van Vleck paramagnetism and its temperature dependence is beyond the scope of this work.

3.4.3. Following $500^{\circ} \mathrm{C}$ annealing. Following $500^{\circ} \mathrm{C}$ annealing, the $M-H$ data are still relatively well reproduced by the model above (figure 10(a)), though clearly not as well as for the as-implanted state. Compared to the as-implanted state, although the fitted $S=2.4(6)$ remains unchanged within the error, the error itself increases significantly (from 0.1 to 0.6$)$. In addition, $N$ decreases to $3.6(6) \times 10^{14}$. This indicates that one or both of the following modifications occurred upon annealing: (1) part of the $\mathrm{Fe}^{3+}$ fraction was converted to $\mathrm{Fe}^{2+}$ due to partial annealing of defects; (2) part of the isolated (magnetically active) Fe impurities suffered short-range diffusion and formed pairs or larger complexes with other Fe impurities (thus contributing with a vanishingly 


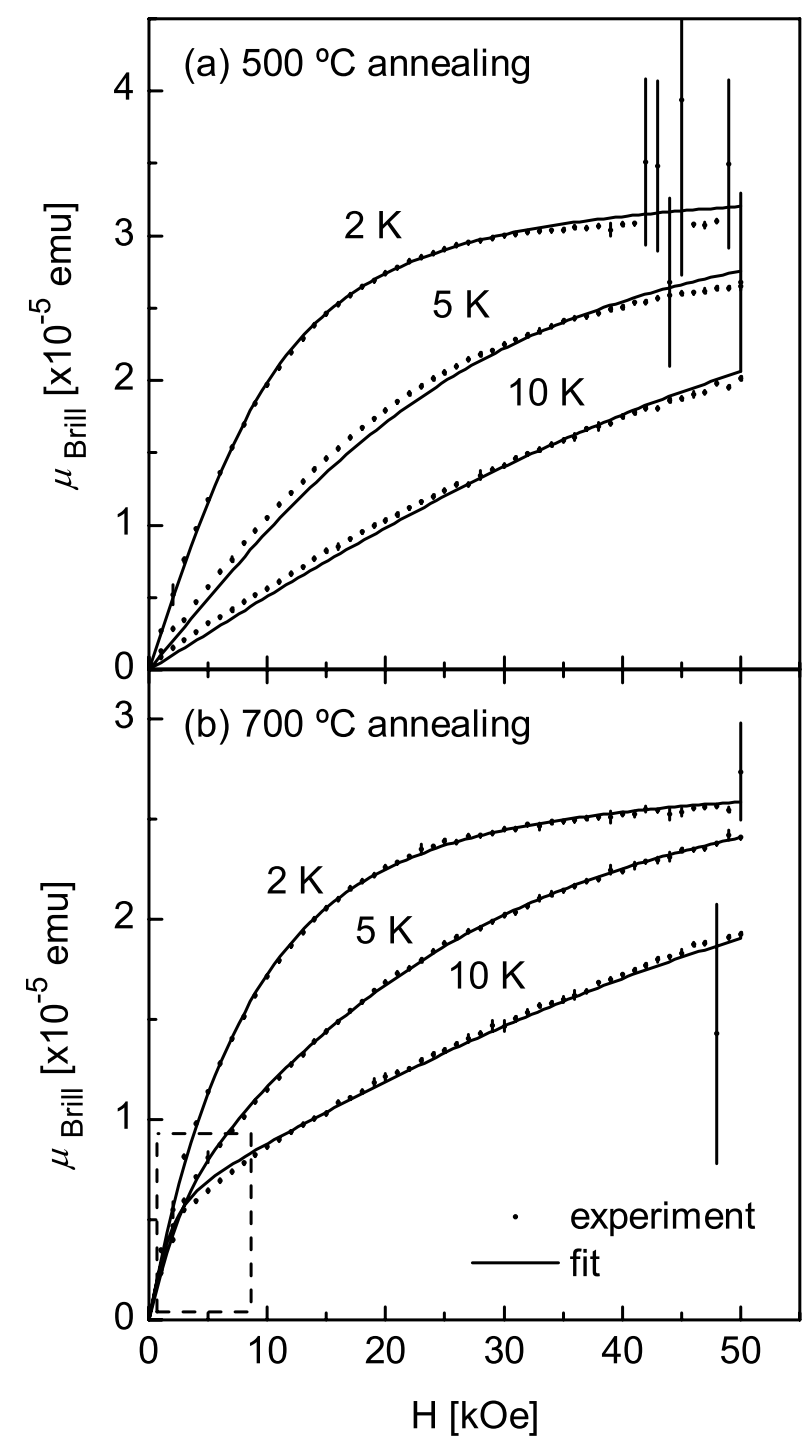

Figure 10. Brillouin-like component of the isothermal $M-H$ data at 2,5 and $10 \mathrm{~K}$ of sample $\mathrm{S} 5$ and corresponding theoretical fits (models described in the text): (a) following $500{ }^{\circ} \mathrm{C}$ annealing; (b) following $700^{\circ} \mathrm{C}$ annealing.

small moment). As we show below, the analysis of the data corresponding to the next annealing step indicates that, in fact, both (1) and (2) occurred. Note that also the van Vleck-like component $\Delta \chi_{\mathrm{vV}}^{\mathrm{ZnFeO}}$ decreased with annealing (figure 9), which is consistent with the decrease in defect density as probed by RBS/C.

3.4.4. Following $700^{\circ} \mathrm{C}$ annealing. Fitting the $700^{\circ} \mathrm{C}$ data with the same model, i.e. with only one $S$ value for the implanted Fe component, gives $S=5.8(4)$, i.e. more than twice the maximum spin-only value of $5 / 2$ for a $3 d$ moment. This indicates the formation of sufficiently large complexes where, although antiferromagnetic interactions may dominate, frustration effects or uncompensated spins result in a non-vanishing net moment. Lacking a better (and still simple) description of such a system, it is worthwhile attempting to fit the data with a model simply based on Brillouin-like paramagnetism. This can be done by allowing
Table 2. Best fit parameters of the Brillouin component of the low-temperature $M-H$ data of sample S5, obtained using the models described in the text.

\begin{tabular}{lllll}
\hline & $S$ & $N\left(\times 10^{14}\right)$ & $S_{2}$ & $N_{2}\left(\times 10^{14}\right)$ \\
\hline As-implanted & $2.5(1)$ & $5.6(4)$ & - & - \\
$500^{\circ} \mathrm{C}$ annealed & $2.4(6)$ & $3.6(6)$ & - & - \\
$700^{\circ} \mathrm{C}$ annealed & $1.8(7)$ & $1.8(7)$ & $9(3)$ & $0.2(1)$ \\
\hline
\end{tabular}

two Brillouin-like fractions with the corresponding $N, N_{2}, S$ and $S_{2}$ free parameters, in addition to $\Delta \chi_{\mathrm{vV}}^{\mathrm{ZnFeO}}$. Figure $10(\mathrm{~b})$ compares the experimental data to the best fit obtained using such a model, i.e. introducing a second Brillouin component with the corresponding $N_{2}$ and $S_{2}$ parameters free at all temperatures. The best fit of the $2 \mathrm{~K}$ data is obtained for $S=$ 1.8(7) and 9(3). For the 5 and $10 \mathrm{~K}$ data, the best fit $S_{2}$ values vary considerably, up to $S=70(15)$, showing that the large- $S$ fraction is in fact very poorly described by the Brillouin function. Nevertheless, this analysis indeed suggests that part of the Fe impurities aggregate in larger complexes. In fact, this can be inferred independently of any fit, from the increase in low-field susceptibility (dashed rectangle in figure 10) without an increase in saturation moment. In addition, the decrease of the small-S component from $S=2.4(6)$ to $S=1.8(7)$ (although with a large error), suggests that part of the isolated $\mathrm{Fe}^{3+} \mathrm{d}^{5}(S=5 / 2)$ fraction may have been converted to $\mathrm{Fe}^{2+} \mathrm{d}^{6}(S=2)$.

Regarding the van Vleck-like component $\Delta \chi_{\mathrm{vV}}^{\mathrm{ZnFO}}$, it is greatly decreased after $700{ }^{\circ} \mathrm{C}$ annealing (figure 9), consistent with further damage recovery. This is discussed in more detail below.

\section{Discussion}

We will now summarize and discuss the magneto-structural analysis presented above, dividing it into four main effects. First and most important, Zn-substitutional Fe impurities in $\mathrm{ZnO}$ are paramagnetic when isolated and interact antiferromagnetically when in nearest-cation-neighbor complexes. Second, under the implantation and annealing conditions that we used, the aggregation of $\mathrm{Zn}$-substitutional Fe impurities in $\mathrm{ZnO}$ occurs without the segregation of a secondary phase. Third, Fe implantation creates a van Vleck-like paramagnetic component which we suggest to originate from the defects created upon irradiation. In a fourth and last point of discussion, we will comment on the absence of ferromagnetism in Fe-implanted $\mathrm{ZnO}$, from the perspective of the various mechanisms of ferromagnetic order in wide-gap DMS proposed so far.

\subsection{Paramagnetism and antiferromagnetic interactions}

Table 2 compiles the parameters obtained from fitting the low temperature $M-H$ data using the model described above. Combined with the $\mathrm{RBS} / \mathrm{C}$ and $\mathrm{EC}$ results, the magneto-structural behavior of $\mathrm{Fe}$ impurities in $\mathrm{ZnO}$ can be summarized as follows. 
Upon implantation, the vast majority of the Fe impurities are more or less randomly incorporated in $\mathrm{Zn}$-substitutional sites (i.e. region (1) of the single-phase region of the phase diagram, figure 1). A significant fraction of these $\mathrm{Fe}$ impurities form impurity-defect complexes with neighboring native point defects, most likely $\mathrm{Zn}$ vacancies (acceptors), assuming a $3+$ charge state and a $\mathrm{d}^{5}(S=5 / 2)$ paramagnetic moment. The formation of such impurity-defect complexes in Fe-implanted $\mathrm{ZnO}$, and the resulting $3+$ charge state of the $\mathrm{Fe}$ impurities has been established by the extensive Mössbauer spectroscopy work of Weyer et al [58-60].

Thermal annealing at moderate temperatures $\left(\sim 500^{\circ} \mathrm{C}\right)$ has two parallel effects on the paramagnetic Fe impurities: (i) thermally activated dissociation of part of the impurity-defect complexes; (ii) the increased mobility of the Fe impurities at moderated temperatures allows part of the Fe impurities to diffuse across short distances and form substitutional $\mathrm{Fe}-\mathrm{Fe}$ dimers. This state can be regarded as an intermediate region between regions (1) and (2) of the single-phase region of the phase diagram, figure 1 . Based on the decrease in $N$ from $5.6(4) \times 10^{14}$ to $3.6(6) \times 10^{14}$, we estimate that about one third of the isolated Fe impurities form dimers, where strong nearest-cation-neighbor antiferromagnetic interactions impose the antiparallel alignment of the Fe spins, resulting in a vanishingly small net magnetic moment per complex. As mentioned above, a similar moment-compensation effect has been observed in other DMS systems, e.g. Co-doped ZnO [27, 28] and Cr-doped GaN [34].

Thermal annealing at higher temperatures $\left(\sim 700^{\circ} \mathrm{C}\right)$ accelerates both (thermally activated) processes (i) and (ii) mentioned in the previous paragraph. The $\mathrm{Zn}$-sublattice defects in the vicinity of the Fe impurities are annealed (i), and the fraction of paramagnetic Fe decreases to a third of that in the as-implanted state (ii), due to Fe aggregation and resulting magnetic compensation. However, unlike after the $500^{\circ} \mathrm{C}$ annealing, the decrease in the isolated $\mathrm{Fe}$ fraction cannot be attributed to dimer formation alone. A high-spin fraction also appears (with $S_{2}$ and $N_{2}$ ), corresponding to about $40 \%$ of the Fe-related magnetization, which we attribute to $\mathrm{Fe}$ aggregates larger than dimers (i.e. with more than two Fe atoms per complex). These aggregates can be regarded as regions of the $\mathrm{Zn}_{1-x} \mathrm{Fe}_{x} \mathrm{O}$ layer where $x$ is significantly larger than $x_{\mathrm{p}}$ and approaches 1. This aggregation state corresponds to region (2) of the single-phase region of the phase diagram, figure 1. In such substitutional Fe aggregates, strong nearest-cation-neighbor antiferromagnetic interactions favor the antiparallel alignment of the Fe spins. However, due the lack of translation symmetry (random $\mathrm{Zn} / \mathrm{Fe}$ cation-site occupation) of such structures as well as their finite size, frustration effects and uncompensated spins result in a non-vanishing net moment per complex.

\subsection{Fe aggregation and segregation mechanisms}

Based on the EC and magnetization data, we have excluded the formation of significant fractions of any magnetic secondary phase from the as-implanted state up to $900^{\circ} \mathrm{C}$ annealing (i.e. we remained in regions (1) and (2) of the single-phase region of the phase diagram, figure 1). This appears to be inconsistent with the findings of Zhou et al [11], who, for similar $\mathrm{Fe}$ concentrations reported that significant fractions of the implanted $\mathrm{Fe}$ impurities segregate in superparamagnetic secondary phases: metallic $\alpha$-Fe nanoprecipitates at processing temperatures of about $500-800{ }^{\circ} \mathrm{C}$ (region (3) in figure 1), and in spinel ferrite $\mathrm{ZnFe}_{2} \mathrm{O}_{4}$ nanoprecipitates at processing temperatures above $\sim 800^{\circ} \mathrm{C}$ (region (4) in figure 1). Instead, in our samples, nearly all Fe impurities $(>70 \%)$ substitute for $\mathrm{Zn}$ in the $\mathrm{ZnO}$ wurtzite structure and, in addition, no superparamagnetic fraction is observed within the sensitivity of our SQUID measurements $(<1 \%)$. In other words, in our samples, thermal annealing simply promoted the aggregation of $\mathrm{Zn}$-substitutional $\mathrm{Fe}$ impurities into Fe-rich regions of the wurtzite $\mathrm{Zn}_{1-x} \mathrm{Fe}_{x} \mathrm{O}$-implanted layer. We suggest that this apparent discrepancy results from different defect accumulation mechanisms. Lattice defects, either point-like or extended, affect the diffusivity of the $\mathrm{Fe}$ impurities and, therefore, may lead to different Fe segregation mechanisms. The differences in defect accumulation, on the other hand, may originate from a combination of (i) different implantation parameters (ion energy, current density, implantation temperature) and (ii) different initial conditions of the implanted $\mathrm{ZnO}$ materials in terms of crystalline quality. Indeed, Zhou et al have shown that the segregation of the implanted $\mathrm{Fe}$ impurities is very sensitive to the $\mathrm{ZnO}$ crystallinity prior to implantation: the formation of $\alpha-\mathrm{Fe}$ clusters $[11,10]$ was suppressed by annealing the $\mathrm{ZnO}$ substrates prior to implantation [9, 12]; bulk ZnO substrates and epitaxial $\mathrm{ZnO}$ thin films showed very distinct segregation behaviors [11].

\section{3. van Vleck-like paramagnetism associated with beam-induced damage}

The fit parameter $\Delta \chi_{\mathrm{vV}}^{\mathrm{ZnFeO}}$, at $2 \mathrm{~K}$, is plotted in figure 11 as a function of annealing temperature, normalized to its maximum value in the as-implanted state. In an attempt to identify from which type (or region) of defects the van Vleck-like paramagnetic component originates, figure 11 compares $\Delta \chi_{\mathrm{vV}}^{\mathrm{ZnFeO}}$ to the bulk and surface minimum yield $\chi_{\text {min }}$ as well as the number of $\mathrm{Fe}^{3+}(N$ in the Brillouin-like fits above), which is in principle a measure of the number of lattice defects involved in the $\mathrm{Fe}^{3+}$-defect complexes. All data sets overlap within the experimental error, which is quite remarkable considering that very different quantities are compared. However, though strongly supporting the defect-related origin of the van Vleck-like susceptibility, this comparison is unable to discern which of the defect components is involved. Further experimental and theoretical work will be necessary in order to elucidate the exact origin of this van Vleck-like paramagnetism.

\subsection{Lack of ferromagnetic order in single-phase $\mathrm{Zn}_{1-x} \mathrm{Fe}_{x} \mathrm{O}$}

Our main finding is essentially the absence of ferromagnetic order in single-phase $\mathrm{Zn}_{1-x} \mathrm{Fe}_{x} \mathrm{O}$, for a wide range of 


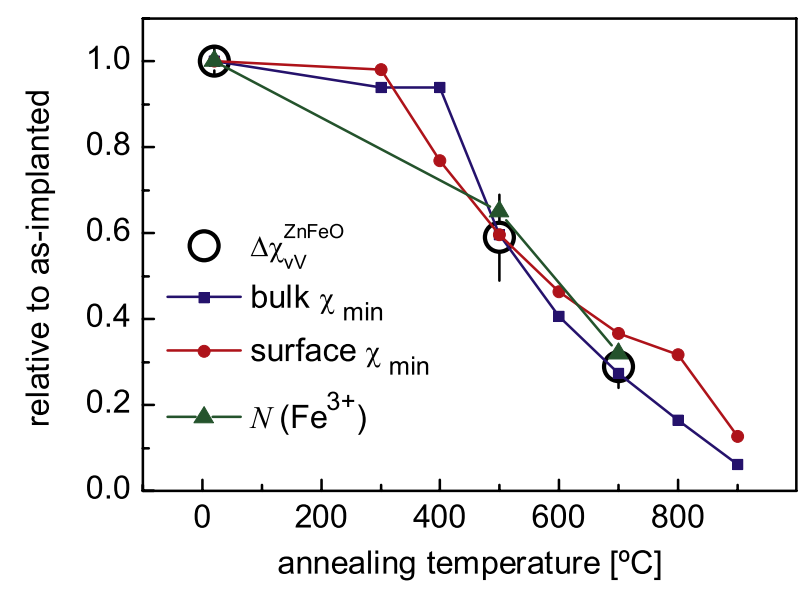

Figure 11. Dependence on annealing temperature of $\Delta \chi_{\mathrm{vV}}^{\mathrm{ZnFeO}}$ at $2 \mathrm{~K}$, compared to the quantities associated with its potential sources, i.e. the quantities associated with the various types (or regions) of defects which may originate the van Vleck-like paramagnetism. Bulk and surface $\chi_{\min }$ are a measure of the defects in the bulk and surface regions, respectively. The number $N$ of Fe ${ }^{3+}$ impurities $(N$ in the Brillouin-like fits) is a measure of the amount of defects involved in the $\mathrm{Fe}^{3+}$-defect complexes. All values are normalized to the respective maximum value, i.e. in the as-implanted state.

Fe concentration $x$ and density of lattice defects. The effects of magnetic interactions can be inferred from the magnetization data, but that interaction is antiferromagnetic and short-ranged. Similar behavior has been observed for $\mathrm{Co}$ impurities in $\mathrm{ZnO}[27,28,31,32]$, which is consistent with the antiferromagnetic nature of the corresponding oxides $\mathrm{FeO}$ and CoO, with Néel temperatures of 198 and 291 K [70], respectively. This suggests that the localized $3 \mathrm{~d}$ moments of $\mathrm{Fe}$ impurities in $\mathrm{ZnO}$ can only interact through indirect superexchange via the $\mathrm{O} 2 \mathrm{p}$ band, thus excluding any of the mechanisms of long-range ferromagnetic order so far proposed for wide-gap DMS. $\mathrm{Zn}_{1-x} \mathrm{Fe}_{x} \mathrm{O}$ being a fairly representative example of wide-gap DMS systems, it is worthwhile discussing why such mechanisms fail to produce ferromagnetic order.

Carrier-mediated ferromagnetism, e.g. via p-d Zener exchange [19], is easily excluded since, unlike Mn in GaAs and InAs, Fe impurities do not introduce the required free carriers in $\mathrm{ZnO}$ (p-holes in the Zener case).

As an alternative to carrier-mediated mechanisms, hightemperature ferromagnetism in wide-gap DMS materials has also been proposed on the basis of bound magnetic polarons (BMP) [36]. Here, the magnetic moments interact via electrons of an impurity band associated with lattice defects, such as those created during implantation. However, the exchange energy density necessary to produce high-temperature ferromagnetism with a few \% of transition metal doping corresponds to a magnetic exchange much stronger than that observed in the strongest known ferromagnetic materials [37]. The lack of such strong magnetic exchange is most likely the reason why we did not observe BMP ferromagnetism down to $2 \mathrm{~K}$, even though we considerably varied the density of beam-induced defects in the $\mathrm{Zn}_{1-x} \mathrm{Fe}_{x} \mathrm{O}$ layers.

A different type of model has been proposed that does not rely on ordered $3 \mathrm{~d}$ local moments and Heisenberg-type exchange as in $\mathrm{p}-\mathrm{d}$ Zener exchange and BMP mechanisms: charge transfer ferromagnetism (CTF) [37], which can be considered a Stoner-type mechanism. CTF requires two distinct types of defects, one that creates a narrow impurity band and another to play the role of a charge reservoir. If both types of defects are present, electrons can be transferred to or from the reservoir until the filling level of the impurity band satisfies the spontaneous spin-split criterion. In dilute magnetic semiconductors, the impurity band can originate, for example, from grain boundaries or beam-induced defects, while mixed-valency impurities may behave as charge reservoirs. However, although $\mathrm{Fe}$ and other $3 \mathrm{~d}$ transition metals have indeed more than one charge state within the $\mathrm{ZnO}$ bandgap, our results suggest that the types of defects produced during implantation do not to satisfy the stringent requirements for the role of the impurity band.

All the mechanisms discussed above ( $\mathrm{p}-\mathrm{d}$ Zener exchange, BMP, CFT and related ones) belong to a general class of collective ferromagnetism and, as such, face a general obstacle in the context of wide-gap DMS: the lack of an efficient mediator of long-range order. An alternative scheme has been proposed in which magnetoelastic effects lead to anisotropy-driven magnetic order in the absence of exchange, i.e. single-ion anisotropy and single-ion magnetism $[71,72]$. Indeed, magnetoelastic spin ordering has been proposed to be the origin of the high-temperature ferromagnetic-like behavior in transition metal doped $\mathrm{SrTiO}_{3}$ [73-76]. In Fe-doped $\mathrm{ZnO}$, on the other hand, such magnetoelastic spin order has not been observed, most likely because the required Jahn-Teller effect is absent or too weak [71]. In $\mathrm{ZnO}, \mathrm{Fe}$ impurities (in $\mathrm{Zn}$ sites, with tetrahedral coordination) are Jahn-Teller ions only if in the 2+ charge state [71], and, even then, $\mathrm{Fe}^{2+}$ ions typically have very low Jahn-Teller energies [77].

\section{Conclusions}

The comprehensive description of wide-gap DMS materials requires a detailed assessment of how dilute magnetic moments behave in a non-magnetic host, from the point of view of structure and magnetism, as well as the correlation between the two. When doing so, one of the main challenges is to identify the growth or doping conditions which lead to single-phase materials, in which the true DMS behavior can be investigated.

We have shown that, in single-phase $\mathrm{Zn}_{1-x} \mathrm{Fe}_{x} \mathrm{O}$ prepared by ion implantation, isolated $\mathrm{Fe}$ impurities occupy $\mathrm{Zn}$ substitutional sites and behave as localized paramagnetic moments down to $2 \mathrm{~K}$, irrespective of Fe concentration and the density of beam-induced defects. With increasing local Fe concentration, short-range antiferromagnetic interactions favor the antiparallel alignment of the localized Fe moments, resulting in an efficient moment compensation.

Together with recent comprehensive studies on singlephase transition metal doped $\mathrm{ZnO}$ and other wide-gap semiconductors, our findings support the view that the absence of collective ferromagnetism is a general feature of wide-gap DMS, resulting from the lack of an efficient 
mediation mechanism for long-range interaction. This notion suggests that the search for high-temperature ferromagnetism in wide-gap DMS may be more successful if exploring single-ion magnetism, such as anisotropy-driven magnetic order induced by magnetoelastic effects.

\section{Acknowledgments}

This work was supported by the Portuguese Foundation for Science and Technology (CERN/FP/123585/2011, SFRH/BD/35761/2007), the European Union Seventh Framework through ENSAR (European Nuclear Science and Applications Research, Contract No. 262010) and SPIRIT (Support of Public and Industrial Research Using Ion Beam Technology, Contract No. 227012), the Fund for Scientific Research-Flanders, and KU Leuven Projects No. GOA/2009/006 and GOA/2014/007.

\section{References}

[1] Dietl T 2010 Nature Mater. 9965

[2] Pereira L M C, Araujo J P, Van Bael M J, Temst K and Vantomme A 2011 J. Phys. D: Appl. Phys. 44215001

[3] Abraham D W, Frank M M and Guha S 2005 Appl. Phys. Lett. 87252502

[4] Ney A, Kammermeier T, Ney V, Ollefs K and Ye S 2008 J. Magn. Magn. Mater. 3203341

[5] Garcia M A, Fernandez Pinel E, de la Venta J, Quesada A, Bouzas V, Fernandez J F, Romero J J, Martin Gonzalez M S and Costa-Kramer J L $2009 \mathrm{~J}$. Appl. Phys. 105013925

[6] Sawicki M, Stefanowicz W and Ney A 2011 Semicond. Sci. Technol. 26064006

[7] Sun Z et al 2008 Phys. Rev. B 77245208

[8] Martinez-Criado G, Somogyi A, Ramos S, Campo J, Tucoulou R, Salome M, Susini J, Hermann M, Eickhoff M and Stutzmann M 2005 Appl. Phys. Lett. 86131927

[9] Potzger K, Zhou S, Reuther H, Kuepper K, Talut G, Helm M, Fassbender J and Denlinger J D 2007 Appl. Phys. Lett. 91062107

[10] Zhou S, Potzger K, Reuther H, Talut G, Eichhorn F, von Borany J, Skorupa W, Helm M and Fassbender J 2007 J. Phys. D: Appl. Phys. 40964

[11] Zhou S et al 2008 J. Appl. Phys. 103023902

[12] Zhou S et al 2008 J. Phys. D: Appl. Phys. 41105011

[13] Zhou S, Potzger K, von Borany J, Grotzschel R, Skorupa W, Helm M and Fassbender J 2008 Phys. Rev. B 77035209

[14] Zhou S, Potzger K, Kuepper K, Grenzer J, Helm M, Fassbender J, Arenholz E and Denlinger J D $2008 \mathrm{~J}$. Appl. Phys. 103043901

[15] Borges R P, Ribeiro B, Costa A R G, Silva C, da Silva R C, Evans G, Goncalves A P, Cruz M M and Godinho M 2011 Eur. Phys. J. B 79185

[16] Ney A, Kovacs A, Ney V, Ye S, Ollefs K, Kammermeier T, Wilhelm F, Rogalev A and Dunin-Borkowski R E 2011 New J. Phys. 13103001

[17] Ney A et al 2010 New J. Phys. 12013020

[18] Matsumoto Y, Murakami M, Shono T, Hasegawa T, Fukumura T, Kawasaki M, Ahmet P, Chikyow T, Koshihara S and Koinuma H 2001 Science 291854

[19] Dietl T, Ohno H, Matsukura F, Cibert J and Ferrand D 2000 Science 2871019

[20] Pearton S J, Abernathy C R, Norton D P, Hebard A F, Park Y D, Boatner L A and Budai J D 2003 Mater. Sci. Eng. R 40137
[21] Prellier W, Fouchet A and Mercey B 2003 J. Phys.: Condens. Matter 15 R1583

[22] Pearton S J, Heo W H, Ivill M, Norton D P and Steiner T 2004 Semicond. Sci. Technol. 19 R59

[23] Liu C, Yun F and Morkoc H 2005 J. Mater. Sci., Mater. Electron. 16555

[24] Stefanowicz W et al 2010 Phys. Rev. B 81235210

[25] Ney A, Kammermeier T, Ollefs K, Ye S, Ney V, Kaspar T C, Chambers S A, Wilhelm F and Rogalev A 2010 Phys. Rev. B 81054420

[26] Granville S, Ruck B J, Budde F, Trodahl H J and Williams G V M 2010 Phys. Rev. B 81184425

[27] Ney A, Ollefs K, Ye S, Kammermeier T, Ney V, Kaspar T C, Chambers S A, Wilhelm F and Rogalev A 2008 Phys. Rev. Lett. 100157201

[28] Sati P, Deparis C, Morhain C, Schafer S and Stepanov A 2007 Phys. Rev. Lett. 98137204

[29] Bonanni A et al 2008 Phys. Rev. Lett. 101135502

[30] Bonanni A et al 2007 Phys. Rev. B 75125210

[31] Xu Q et al 2009 J. Phys. D: Appl. Phys. 42085001

[32] de Carvalho H B et al 2010 J. Appl. Phys. 108079906

[33] Bonanni A et al 2011 Phys. Rev. B 84035206

[34] Pereira L M C, Som T, Demeulemeester J, Van Bael M J, Temst K and Vantomme A 2011 J. Phys.: Condens. Matter 23346004

[35] Sarigiannidou E, Wilhelm F, Monroy E, Galera R M, Bellet-Amalric E, Rogalev A, Goulon J, Cibert J and Mariette H 2006 Phys. Rev. B 74041306

[36] Coey J M D, Venkatesan M and Fitzgerald C B 2005 Nature Mater. 4173

[37] Coey J M D, Stamenov P, Gunning R D, Venkatesan M and Paul K 2010 New J. Phys. 12053025

[38] Potzger K et al 2006 Appl. Phys. Lett. 88052508

[39] Wu P et al 2006 Appl. Phys. Lett. 89012508

[40] Song Y Y, Park K S, Son D V, Yu S C, Kang H J, Shin S W, Whang C N, Lee J H, Song J H and Lee K W 2007 J. Korean Phys. Soc. 501706

[41] Kumar R, Singh A P, Thakur P, Chae K H, Choi W K, Angadi B, Kaushik S D and Patnaik S 2008 J. Phys. D: Appl. Phys. 41155002

[42] Robinson M T 1989 Phys. Rev. B 4010717

[43] Chu W K, Mayer J W and Nicolet M A 1978 Backscattering Spectrometry (New York: Academic)

[44] Hofsäss H and Lindner G 1991 Phys. Rep. 201121

[45] Hofsäss H, Wahl U and Jahn S G 1994 Hyperfine Interact. 8427

[46] Hofsäss H 1996 Hyperfine Interact. 97247

[47] Wahl U 2000 Hyperfine Interact. 129349

[48] Rita E, Wahl U, Correia J G, Alves E and Soares J C 2004 Appl. Phys. Lett. 85 4899-901

[49] Pereira L M C, Wahl U, Decoster S, Correia J G, Amorim L M, da Silva M R, Araujo J P and Vantomme A 2011 Phys. Rev. B 84125204

[50] Wahl U, Rita E, Correia J G, Alves E and Soares J G 2004 Phys. Rev. B 69012102

[51] Fedoseyev V N et al 1997 Nucl. Instrum. Methods Phys. Res. B 12688

[52] Wahl U, Correia J G, Cardoso S, Marques J G, Vantomme A, Langouche G and ISOLDE Collaboration $1998 \mathrm{Nucl}$. Instrum. Methods Phys. Res. B 136744

[53] Agostinelli S et al 2003 Nucl. Instrum. Methods Phys. Res. A 506250

[54] Allison J et al 2006 IEEE Trans. Nucl. Sci. 53270

[55] Kucheyev S O, Williams J S, Jagadish C, Zou J, Evans C, Nelson A J and Hamza A V 2003 Phys. Rev. B 67094115

[56] Ambacher O 1998 J. Phys. D: Appl. Phys. 312653

[57] Wahl U, Rita E, Correia J G, Alves E, Araujo J P and ISOLDE Collaboration 2003 Appl. Phys. Lett. 821173 
[58] Weyer G, Gunnlaugsson H P, Mantovan R, Fanciulli M, Naidoo D, Bharuth-Ram K and Agne T 2007 J. Appl. Phys. 102113915

[59] Gunnlaugsson H P et al 2010 Appl. Phys. Lett. 97142501

[60] Molholt T E et al 2009 Physica B 4044820

[61] Azamat D V and Fanciulli M 2007 Physica B 401382

[62] Khalid M, Setzer A, Ziese M, Esquinazi P, Spemann D, Poeppl A and Goering E 2010 Phys. Rev. B 81214414

[63] Ney A, Harris J S Jr and Parkin S S P 2006 J. Phys.: Condens. Matter 184397

[64] Maeda K, Sato M, Niikura I and Fukuda T 2005 Semicond Sci. Technol. 20 S49

[65] Heitz R, Hoffmann A and Broser I 1992 Phys. Rev. B 458977

[66] Chikoidze E, von Bardeleben H J, Dumont Y, Galtier P and Cantin J L 2005 J. Appl. Phys. 97 10D316

[67] Heitz R, Thurian P, Loa I, Eckey L, Hoffmann A, Broser I, Pressel K, Meyer B K and Mokhov E N 1995 Appl. Phys. Lett. 672822
[68] Marcet S, Ferrand D, Halley D, Kuroda S, Mariette H, Gheeraert E, Teran F J, Sadowski M L, Galera R M and Cibert J 2006 Phys. Rev. B 74125201

[69] Behringer R E 1958 J. Chem. Phys. 29537

[70] Kittel C 2004 Introduction to Solid State Physics 8th edn (New York: Wiley)

[71] Dionne G F 2007 J. Appl. Phys. 101 09C509

[72] Dionne G F and Kim H-S 2008 J. Appl. Phys. 103 07B333

[73] Bi L, Kim H-S, Dionne G F and Ross C A 2010 New J. Phys. 12043044

[74] Jiang P, Bi L, Kim D H, Dionne G F and Ross C A 2011 Appl. Phys. Lett. 98231909

[75] Kim D H, Bi L, Jiang P, Dionne G F and Ross C A 2011 Phys. Rev. B 84014416

[76] Kim H-S, Bi L, Kim D H, Yang D-J, Choi Y J, Lee J W, Kang J K, Park Y C, Dionne G F and Ross C A 2011 J. Mater. Chem. 21 10364-9

[77] Goodenough J B 1964 J. Phys. Chem. Solids 25151 